\title{
Psychological therapies for the treatment of anxiety disorders in chronic obstructive pulmonary disease (Review)
}

Usmani ZA, Carson KV, Heslop K, Esterman AJ, De Soyza A, Smith BJ

Usmani ZA, Carson KV, Heslop K, Esterman AJ, De Soyza A, Smith BJ.

Psychological therapies for the treatment of anxiety disorders in chronic obstructive pulmonary disease.

Cochrane Database of Systematic Reviews 2017, Issue 3. Art. No.: CD010673.

DOI: 10.1002/14651858.CD010673.pub2.

www.cochranelibrary.com

Psychological therapies for the treatment of anxiety disorders in chronic obstructive pulmonary disease 
TABLE OF CONTENTS

HEADER 1

ABSTRACT

PLAIN LANGUAGE SUMMARY

SUMMARY OF FINDINGS

BACKGROUND

OBJECTIVES

METHODS

RESULTS

Figure 1.

Figure 2.

Figure 3.

Figure 4.

DISCUSSION

AUTHORS' CONCLUSIONS

ACKNOWLEDGEMENTS

REFERENCES

CHARACTERISTICS OF STUDIES

DATA AND ANALYSES

Analysis 1.1. Comparison 1 Psychological therapies versus co-intervention, Outcome 1 Anxiety.

Analysis 1.2. Comparison 1 Psychological therapies versus co-intervention, Outcome 2 Quality of life.

Analysis 1.3. Comparison 1 Psychological therapies versus co-intervention, Outcome 3 Six minute walking distance. .............

Analysis 2.1. Comparison 2 Duration of intervention sub-group analyses - psychological therapies versus co-intervention, Outcome 1 Anxiety.

Analysis 2.2. Comparison 2 Duration of intervention sub-group analyses - psychological therapies versus co-intervention, Outcome 2 Six minute walking distance.

Analysis 2.3. Comparison 2 Duration of intervention sub-group analyses - psychological therapies versus co-intervention, Outcome 3 Quality of life - SGRQ and SF36 Physical composite.

Analysis 2.4. Comparison 2 Duration of intervention sub-group analyses - psychological therapies versus co-intervention, Outcome 4 Quality of life - SGRQ and SF36 Emotional composite.

APPENDICES

CONTRIBUTIONS OF AUTHORS

DECLARATIONS OF INTEREST

SOURCES OF SUPPORT

DIFFERENCES BETWEEN PROTOCOL AND REVIEW

INDEX TERMS 
[Intervention Review]

\section{Psychological therapies for the treatment of anxiety disorders in chronic obstructive pulmonary disease}

Zafar A Usmani1,2, Kristin V Carson², Karen Heslop³, Adrian J Esterman4, Anthony De Soyza5 ${ }^{5}$ Brian J Smith²

1Department of Respiratory Medicine, The Queen Elizabeth Hospital, Adelaide, Australia. 2School of Medicine, The University of Adelaide, Adelaide, Australia. ${ }^{3}$ Chest Clinic, Newcastle upon Tyne NHS Hospitals Foundation Trust, Newcastle upon Tyne, UK. ${ }^{4}$ Division of Health Sciences, University of South Australia, Adelaide, Australia. ${ }^{5}$ Institute of Cellular Medicine, Newcastle University, Newcastle, UK

Contact address: Zafar A Usmani, Department of Respiratory Medicine, The Queen Elizabeth Hospital, 4A, Main Building, 28 Woodville Road, Woodville South, Adelaide, SA 5011, Australia. zafar-ahmad.usmani@health.sa.gov.au.

Editorial group: Cochrane Common Mental Disorders Group. Publication status and date: New, published in Issue 3, 2017.

Citation: Usmani ZA, Carson KV, Heslop K, Esterman AJ, De Soyza A, Smith BJ. Psychological therapies for the treatment of anxiety disorders in chronic obstructive pulmonary disease. Cochrane Database of Systematic Reviews 2017, Issue 3. Art. No.: CD010673. DOI: 10.1002/14651858.CD010673.pub2.

Copyright $(2017$ The Cochrane Collaboration. Published by John Wiley \& Sons, Ltd.

\section{A B S T R A C T}

\section{Background}

Chronic obstructive pulmonary disease (COPD) (commonly referred to as chronic bronchitis and emphysema) is a chronic lung condition characterised by the inflammation of airways and irreversible destruction of pulmonary tissue leading to progressively worsening dyspnoea. It is a leading international cause of disability and death in adults. Evidence suggests that there is an increased prevalence of anxiety disorders in people with COPD. The severity of anxiety has been shown to correlate with the severity of COPD, however anxiety can occur with all stages of COPD severity. Coexisting anxiety and COPD contribute to poor health outcomes in terms of exercise tolerance, quality of life and COPD exacerbations. The evidence for treatment of anxiety disorders in this population is limited, with a paucity of evidence to support the efficacy of medication-only treatments. It is therefore important to evaluate psychological therapies for the alleviation of these symptoms in people with COPD.

\section{Objectives}

To assess the effects of psychological therapies for the treatment of anxiety disorders in people with chronic obstructive pulmonary disease.

\section{Search methods}

We searched the specialised registers of two Cochrane Review Groups: Cochrane Common Mental Disorders (CCMD) and Cochrane Airways (CAG) (to 14 August 2015). The specialised registers include reports of relevant randomised controlled trials from The Cochrane Library, MEDLINE, Embase, and PsycINFO. We carried out complementary searches on PsycINFO and CENTRAL to ensure no studies had been missed. We applied no date or language restrictions.

\section{Selection criteria}

We considered all randomised controlled trials (RCTs), cluster-randomised trials and cross-over trials of psychological therapies for people (aged over 40 years) with COPD and coexisting anxiety disorders (as confirmed by recognised diagnostic criteria or a validated measurement scale), where this was compared with either no intervention or education only. We included studies in which the psychological therapy was delivered in combination with another intervention (co-intervention) only if there was a comparison group that received the co-intervention alone. 


\section{Data collection and analysis}

Two review authors independently screened citations to identify studies for inclusion and extracted data into a pilot-tested standardised template. We resolved any conflicts that arose through discussion. We contacted authors of included studies to obtain missing or raw data. We performed meta-analyses using the fixed-effect model and, if we found substantial heterogeneity, we reanalysed the data using the random-effects model.

\section{Main results}

We identified three prospective RCTs for inclusion in this review (319 participants available to assess the primary outcome of anxiety). The studies included people from the outpatient setting, with the majority of participants being male. All three studies assessed psychological therapy (cognitive behavioural therapy) plus co-intervention versus co-intervention alone. We assessed the quality of evidence contributing to all outcomes as low due to small sample sizes and substantial heterogeneity in the analyses. Two of the three studies had prespecified protocols available for comparison between prespecified methodology and outcomes reported within the final publications.

We observed some evidence of improvement in anxiety over 3 to 12 months, as measured by the Beck Anxiety Inventory (range from 0 to 63 points), with psychological therapies performing better than the co-intervention comparator arm (mean difference (MD) -4.41 points, $95 \%$ confidence interval $(\mathrm{Cl})-8.28$ to $-0.53 ; \mathrm{P}=0.03)$. There was however, substantial heterogeneity between the studies $(12=62 \%)$, which limited the ability to draw reliable conclusions. No adverse events were reported.

\section{Authors' conclusions}

We found only low-quality evidence for the efficacy of psychological therapies among people with COPD with anxiety. Based on the small number of included studies identified and the low quality of the evidence, it is difficult to draw any meaningful and reliable conclusions. No adverse events or harms of psychotherapy intervention were reported.

A limitation of this review is that all three included studies recruited participants with both anxiety and depression, not just anxiety, which may confound the results. We downgraded the quality of evidence in the 'Summary of findings' table primarily due to the small sample size of included trials. Larger RCTs evaluating psychological interventions with a minimum 12-month follow-up period are needed to assess long-term efficacy.

\section{PLAIN LANGUAGE SUMMARY}

Psychotherapy for treatment of anxiety in chronic obstructive pulmonary disease (chronic bronchitis and emphysema)

\section{Why is this review important?}

Chronic obstructive pulmonary disease (COPD) is commonly referred to as emphysema and chronic bronchitis. People with COPD are more likely to have anxiety disorders compared with the general population. Symptoms of anxiety affect various aspects of daily life, including quality of life and the ability to perform physical activities. Psychological therapies are used as part of clinical practice to treat these symptoms, however, there is little evidence to support these techniques.

\section{Who will be interested in this review?}

Health professionals and people with emphysema and underlying anxiety and panic.

\section{What questions does this review aim to answer?}

What is the current evidence on psychological therapies for anxiety in people with COPD and coexisting anxiety?

\section{Which studies were included in the review?}

Randomised controlled trials (research trials in which participants are allocated according to a random sequence either to the intervention to be tested or to a comparator intervention).

\section{What does the evidence from the review tell us?}

This systematic review found three studies with a total of 319 participants with COPD and coexisting anxiety. All three studies assessed psychotherapy (CBT) with a co-intervention, versus the co-intervention alone. There was limited evidence showing some improvements in reduced levels of anxiety and improved quality of life in the psychotherapy group. It is important to note that the overall quality of the evidence was low and hence further research is needed to increase our confidence in this effect. A limitation of this review is that all three included studies recruited participants with both anxiety and depression, not just anxiety, which may confound the results.

\section{What should happen next?}


Further research is needed to establish whether this therapy will reduce hospital admissions and length of hospital stays, as this was not assessed in the current evidence base. Larger studies of longer duration need to be conducted. There are at least two more clinical trials currently ongoing for this question. Once they are published, the evidence from them could increase or decrease our confidence in the findings of this review. 


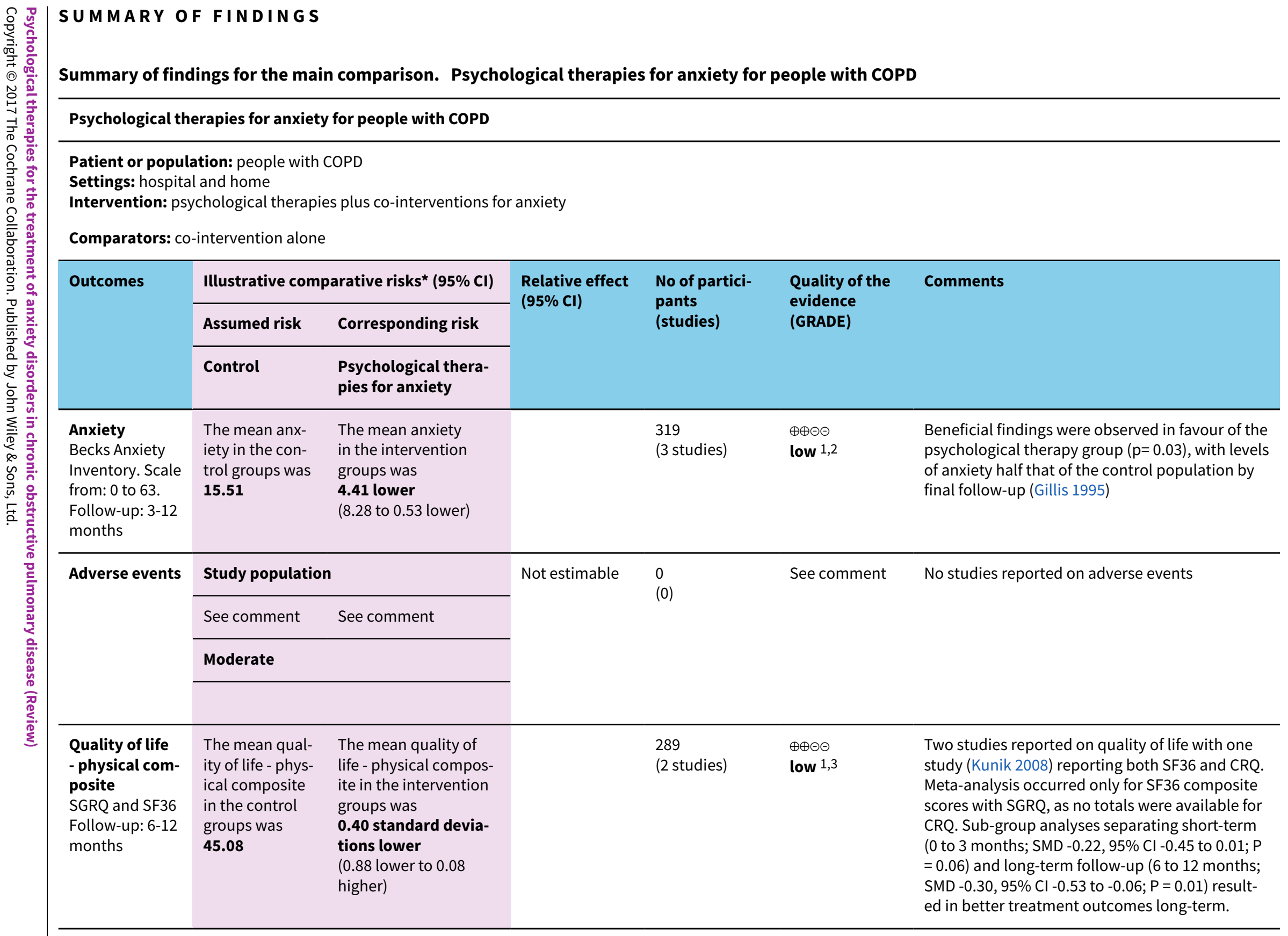

Settings: hospital and home

Comparators: co-intervention alone 


\begin{tabular}{|c|c|c|c|c|c|}
\hline $\begin{array}{l}\text { Quality of life } \\
\text { - emotional } \\
\text { composite } \\
\text { SGRQ and SF36 } \\
\text { Follow-up: 6-12 } \\
\text { months }\end{array}$ & $\begin{array}{l}\text { The mean qual- } \\
\text { ity of life - emo- } \\
\text { tional compos- } \\
\text { ite in the con- } \\
\text { trol groups was } \\
\mathbf{5 2 . 3}\end{array}$ & $\begin{array}{l}\text { The mean quality of } \\
\text { life - emotional com- } \\
\text { posite in the interven- } \\
\text { tion groups was } \\
\mathbf{0 . 3 0} \text { standard devia- } \\
\text { tions lower } \\
\text { (1.03 lower to } 0.44 \\
\text { higher) }\end{array}$ & $\begin{array}{l}289 \\
\text { (2 studies) }\end{array}$ & $\begin{array}{l}\oplus \oplus \ominus \ominus \\
\text { low } 1,3\end{array}$ & $\begin{array}{l}\text { Two studies reported on quality of life with one } \\
\text { study (Kunik 2008) reporting both SF36 and CRQ. } \\
\text { We only meta-analysed SF } 36 \text { composite scores } \\
\text { with SGRQ as no totals were available for CRQ. } \\
\text { Sub-group analyses separating short-term ( } 0 \text { to } \\
3 \text { months; (SMD } 0.05,95 \% \mathrm{Cl}-0.18 \text { to } 0.28 \text { ) and } \\
\text { long-term follow-up ( } 6 \text { to } 12 \text { months; (SMD - } 0.09 \text {, } \\
95 \% \mathrm{Cl}-0.32 \text { to } 0.14 \text { ) resulted in better treatment } \\
\text { outcomes long-term. }\end{array}$ \\
\hline $\begin{array}{l}\text { Exercise ca- } \\
\text { pacity } \\
6 M W D \\
\text { Follow-up: 3-12 } \\
\text { months }\end{array}$ & $\begin{array}{l}\text { The mean ex- } \\
\text { ercise capacity } \\
\text { in the control } \\
\text { groups was } \\
\mathbf{8 3 9}\end{array}$ & $\begin{array}{l}\text { The mean exercise ca- } \\
\text { pacity in the interven- } \\
\text { tion groups was } \\
\mathbf{2 . 7 8} \text { lower } \\
\text { ( } 58.49 \text { lower to } 52.94 \\
\text { higher) }\end{array}$ & $\begin{array}{l}268 \\
\text { (2 studies) }\end{array}$ & $\begin{array}{l}\oplus \oplus \ominus \ominus \\
\text { low } 1,4\end{array}$ & $\begin{array}{l}\text { The Kunik } 2008 \text { study which examined 6MWD } \\
\text { at } 8 \text { weeks (post-intervention) and again at } 12 \\
\text { months' follow-up with a difference in favour of } \\
\text { the control arm at } 12 \text { months }(P=0.05) \text {. However, } \\
\text { authors reported that group means at beginning } \\
\text { of the follow-up period were not equal }(P<0.01) \text {, } \\
\text { contributing to the spurious finding. }\end{array}$ \\
\hline
\end{tabular}

*The basis for the assumed risk (e.g. the median control group risk across studies) is provided in footnotes. The corresponding risk (and its $95 \%$ confidence interval) is based on the assumed risk in the comparison group and the relative effect of the intervention (and its $95 \% \mathrm{Cl}$ ).

6MWD: six minute walking distance; CI: confidence interval; CRQ: Chronic Respiratory Questionnaire; SF36: Short Form 36; SGRQ: Saint George's Respiratory Questionnaire

GRADE Working Group grades of evidence

High quality: further research is very unlikely to change our confidence in the estimate of effect.

Moderate quality: further research is likely to have an important impact on our confidence in the estimate of effect and may change the estimate.

Low quality: further research is very likely to have an important impact on our confidence in the estimate of effect and is likely to change the estimate.

Very low quality: we are very uncertain about the estimate.

1Substantial heterogeneity as identified via the I-squared statistic and visual inspection of the data.

2Two of the three studies have sample sizes lower than the prespecified optimal sample size, study participants were predominantly men across all three studies and most subjects had comorbid anxiety and depression, therefore imprecision was downgraded by one point.

${ }^{3}$ Both studies had sample sizes lower than the prespecified optimal sample size.

4 Wide confidence intervals around effect estimate. 


\section{B A C K G R O U N D}

\section{Description of the condition}

Chronic obstructive pulmonary disease (COPD) is a chronic lung condition characterised by the inflammation of airways and irreversible destruction of pulmonary tissue leading to progressively worsening dyspnoea and is a leading international cause of disability and death in adults (Kerstjens 2001). COPD is a preventable and treatable disease with some significant extrapulmonary effects. Its pulmonary component is characterised by airflow limitation that is not fully reversible (GOLD 2013). The diagnosis of COPD is based on the person's history, evaluation of risk factors and relevant investigations, including pulmonary function testing and chest imaging. Spirometry will typically show a post bronchodilator forced expiratory volume $1 /$ forced vital capacity (FEV1/FVC) less than 70\% (Rabe 2007). Anxiety disorder is a generalised term for a myriad of abnormal and pathological fear and anxiety states, including generalised anxiety disorder (GAD), panic disorder (PD), agoraphobia, neurocirculatory asthenia, obsessive-compulsive disorder (OCD), and phobic disorders. GAD is defined as excessive anxiety which lasts for at least six months. Individuals must also experience three or more of the following symptoms: difficulty in concentrating; fatigue after little exertion; sleep disturbance; a sensation of being 'keyed up' (nervous or anxious); irritability or muscle tension, or both (Diagnostic and Statistical Manual (DSM) IV criteria) (APA 1994). Other anxiety symptoms can include increased behavioural and psychological symptoms of distress (Suh 2013) and fears (Breland 2015), which is of particular importance as disease-specific fears can have an impact on disability (Keil 2014).

Evidence suggests that there is an increased prevalence of anxiety disorders in people with COPD (Maurer 2008). Moreover, the severity of anxiety has been shown to correlate with the severity of COPD and the presence of lower $\mathrm{PaO}_{2}$ (partial pressure of oxygen in the blood, an indicator of severity of COPD)(Elassal 2014). Other studies suggest that anxiety can occur at any stage of COPD (Kim 2000; Heslop-Marshall 2014. Studies have suggested prevalence rates for anxiety disorders of $28 \%-36 \%$ in people with COPD (Di Marco 2006; Yohannnes 2006). The lifetime prevalence of GAD in particular amongst people with COPD is estimated at between 10\% and $15.8 \%$ (Brenes 2003). The prevalence of panic disorder in the COPD population is estimated to be ten times higher than the general population (Smoller 1996; Smoler 1999). Rates of anxiety symptoms in people with COPD range from $13 \%$ to $51 \%$ and are higher than the rates in people with heart failure, cancer, and other medical conditions (Brenes 2003). COPD is associated with a higher risk of anxiety, and co morbid COPD and anxiety is related to poor health outcomes in terms of exercise tolerance, quality of life, COPD exacerbations (Eisner 2010), inappropriate use of medications and persistence of smoking as a coping strategy for anxiety management (Royal College of Physcians). Several risk factors have been identified that contribute to anxiety amongst people with COPD, including being employed, less education, lack of contentment with family support, living with family and friends, comorbid hypertension and depression, and having 10 or more exacerbations per year (Tan 2013). Indeed, co-morbid depression is known to be a strong predictor of anxiety (DiNicola 2013), which confounds anxiety symptoms making treatment more difficult (Atlantis 2013). By compromising health status, mood disorders lead to increased risk of hospitalisation and re-hospitalisation
(Gudmundsson 2005) and hence also increase direct and indirect costs to the health system.

Various models could be considered to explain increased levels of anxiety and panic in people with COPD (Ley 1985; Perna 2004). One model explains this relationship as catastrophic misinterpretations of ambiguous bodily sensations (such as shortness of breath, rapid heart rate) which increase arousal, creating a positive feedback loop that results in a panic attack (Clark 1986). A crucial difference between physically healthy people and those with COPD is that in the latter, breathing, the most basic of all physical functions necessary for life, is objectively threatened (as measured by tests of lung function) and subjectively difficult. Dyspnoea (shortness of breath) can be an unpleasant and potentially frightening experience at any time, and, as the key symptom of an eventually fatal illness like COPD, it is an ambiguous sensation open to catastrophic interpretation, leading to increased levels of anxiety and panic in people with COPD (Livermore 2010b).

\section{Description of the intervention}

Management strategies for the treatment of anxiety disorders in people with COPD include both pharmacological and nonpharmacological interventions. Evidence that pharmacological therapies (anti-anxiety or antidepressant medications, or both) provide statistically or clinically significant benefits for this group of patients is limited (Usmani 2011). Psychological therapies include cognitive or behavioural therapies, or both, psychodynamic psychotherapy, interpersonal psychotherapy, non-directive therapy, support therapy and counselling (Rose 2002; Davison 2003). Psychological therapies are intentional interpersonal relationships used by trained psychotherapists to aid people with problems of living, with an aim of increasing the individual's well-being. Psychological therapies may also be performed by practitioners with a number of different qualifications, including psychologists, marriage and family therapists, occupational therapists, licensed clinical social workers, counsellors, psychiatric nurses, psychotherapists, trained general nurses, psychoanalysts, and psychiatrists. The mode of delivery for these therapies may comprise individual, group or family (including couple) therapy, performed by a healthcare professional.

\section{How the intervention might work}

Because COPD is an irreversible condition, treatment recommendations are aimed at improving quality of life (Norweg 2005). Current evidence examining quality of life suggests a reduction in satisfaction above and beyond what should be expected by COPD disease severity or co-morbid medical illnesses (Coventry 2007), indicating that psychological status plays an intrinsic role in overall well-being. A recent study examining the impact of anxiety on the lives of people with COPD found that they felt isolated and would avoid social occasions and usual daily activities (Willgoss 2011). As a result, therapies targeting the reduction of psychological stressors should be expected to improve quality of life (Ries 1995; Rose 2002; Baraniak 2011).

Psychological therapies are often based on the assumption that psychological outcomes such as anxiety are linked with physical manifestations of COPD, for example dyspnoea, which can precipitate episodes of anxiety (Wu 2004). It has been hypothesised that a person's fear and misinterpretation of bodily experiences from dyspnoea and hyperventilation may cause a panic reaction 
(Nutt 1999). Alternatively, underlying psychological distress can contribute to an increased risk of symptom exacerbations, particularly those treated in the person's own environment (Laurin 2011). As such, people with anxiety and panic disorders interpret threats as more dangerous due to a higher awareness of cues such as dyspnoea and tachycardia (Mikkelsen 2004).

A psychological therapy, cognitive-behaviour therapy (CBT), aims to identify and correct dysfunctional emotions, behaviours and cognitions through a goal-orientated, systematic procedure (Rose 2002; Kaplan 2009). In the case of people with COPD, CBT may be a means of managing concurrent anxiety and depression. While not in itself improving an individual's medical condition, CBT may serve to increase perceived self-efficacy and motivate people to manage their physical condition, thereby improving quality of life (Kunik 2001). Moreover, the learning about oneself that occurs in various forms of psychological therapy may in itself influence the structure and function of the brain (Kandel 1998) or may have a significant impact on serotonin metabolism (Viinamaki 1998). 'Third wave CBT' applies to behavioural psychological therapies that integrate mindfulness and acceptance of unwanted thoughts and feelings with a behavioural understanding of emotional suffering, to elicit change in thinking process. Behavioural therapy includes methods that focus on behaviours, not the thoughts and feelings that might be causing them. The behavioural approach to therapy assumes that behaviour that is associated with psychological problems develops through the same processes of learning that affect the development of other behaviours. Psychodynamic therapy focuses on unconscious processes as they are manifested in people's present behaviour. Hence by making the unconscious aspects of their life a part of their present experience, psychodynamic therapy helps people understand how their behaviour and mood are affected by unconscious feelings. Humanistic psychotherapy emphasises human uniqueness, positive qualities, and individual potential. It works by emphasising one's capacity to make informed and rational choices and develop to one's maximum potential. Integrative therapies are approaches that combine components of different psychological therapy models.

\section{Why it is important to do this review}

Anxiety disorders in people with COPD have been shown to increase disability and impair functional status, resulting in an overall reduction in their quality of life (Beck 1988; Weaver 1997). Importantly, the impact of anxiety on these outcomes was shown after adjusting for other potential confounders such as general health status, other medical conditions and COPD severity (Brenes 2003). Kim 2000 reported that anxiety and depression were more strongly related to functional status than the severity of COPD. Screening data from a large randomised controlled trial in the UK showed anxiety was common in COPD and was not correlated with COPD severity (Heslop-Marshall 2014). Co-morbid anxiety in an elderly population with COPD has been suggested as a significant predictor of the frequency of hospital admissions (Yohannes 2000). A recent study has shown that among people with COPD, anxiety is related to poorer health outcomes including worse submaximal exercise performance, greater risk of self-reported functional limitations and a higher longitudinal risk of COPD exacerbations (Eisner 2010).

The evidence for treatment of anxiety disorders in COPD is limited, and there are limited data to support the efficacy of medicationonly treatments (Borson 1998). The results of a Cochrane Review evaluating the effects of pharmacological interventions for anxiety in people with COPD are inconclusive (Usmani 2011). A feasibility study of antidepressants in this population suggested poor acceptance of antidepressants for various reasons including sideeffects and reluctance to take "yet another medication" (Yohannes 2001). Furthermore the association between anxiety/panic and dyspnoea/COPD had been explained by various psychological theories (Clark 1986; Livermore 2010b). It is important therefore, to evaluate psychological therapies for the alleviation of these symptoms in people with COPD.

In light of the health burden caused by psychological disorders and the limited evidence supporting treatment options, this review is one of four linked Cochrane Reviews that assess the effects of pharmacological and psychological therapies for the treatment of anxiety and depression in people with COPD, one of which has already been published (Usmani 2011).

\section{O B J E C T IVES}

To assess the effects of psychological therapies for the treatment of anxiety disorders in people with chronic obstructive pulmonary disease.

\section{METHODS}

\section{Criteria for considering studies for this review}

\section{Types of studies}

Randomised controlled trials (RCTs), including cross-over trials and cluster-randomised trials.

\section{Types of participants}

Participants were adults over 40 years of age (as clinically significant COPD is generally seen in people more than 40 years of age (GOLD 2013)) of either gender and of any ethnicity, diagnosed with COPD and a recognised anxiety disorder or anxiety symptom(s).

The COPD diagnosis needed to have been made objectively, for example, according to GOLD (Global initiative for chronic Obstructive Lung Disease) criteria, or similar criteria (e.g. FEV1/FVC less than 0.70).

The anxiety disorder (e.g. generalised anxiety disorder (GAD), panic disorder (PD), agoraphobia, neurocirculatory asthenia, obsessivecompulsive disorder (OCD), phobic disorders) needed be defined either using established diagnostic criteria, for example, DSM criteria (APA 1994) or anxiety symptoms identified using a formal psychological instrument, for example, Beck Anxiety Inventory (BAI) (Beck 1961; BAI 1993) or Hospital Anxiety \& Depression Scale (Zigmond 1983) at the time of recruitment to the trial.

We included participants with co-morbid mental health disorders. Anxiety did not need to be the primary mental health disorder for included participants as long as they had formally diagnosed or symptomatic anxiety (as diagnosed or assessed by a formal criteria or a validated tool).

We excluded studies that only assessed psychological therapies for the treatment of depression in people with COPD, as these will be covered by a separate review. 


\section{Types of interventions}

We included studies assessing any form of psychological therapy for the treatment of anxiety disorders in people with COPD where this was compared with either no intervention or education only. Studies in which the psychological therapy was delivered in combination with another intervention (co-intervention) were included only if there was a comparison group that received the cointervention alone.

\section{Experimental interventions:}

- Cognitive behavioural therapy (CBT) (e.g. problem solving, rational emotive therapy)

- Third Wave CBT (i.e. acceptance and commitment therapy, compassionate mind training, functional analytic psychotherapy, mindfulness-based cognitive therapy, behavioural activation, meta-cognitive therapy and dialectical behavioural therapy)

- Behavioural therapy (e.g. behaviour modification, assertiveness training)

- Psychodynamic therapy (e.g. insight-oriented therapy, group psychotherapy)

- Humanistic therapy (e.g. expressive therapy, supportive therapy)

- Integrative therapy (e.g. cognitive analytical therapy)

Comparators:

- No intervention (i.e. waiting list and usual care)

- Education only (education (written or oral), such as provision of information about physical and mental health issues during a medical consultation or during a visit with a nurse where no formal counselling or psychological therapy was provided)

- Co-intervention (only if the same co-intervention was used in the intervention arm of the study). The co-interventions considered were pharmacotherapy and pulmonary rehabilitation.

\section{Types of outcome measures}

\section{Primary outcomes}

- Change in anxiety symptoms as measured by a standardised or validated anxiety measure, for example, State-Trait Anxiety Inventory (STAI) (Spielberger 1970), the Hospital Anxiety and Depression Scale (HADS) (Zigmond 1983) and the Beck Anxiety Inventory (BAI) (Beck 1961; BAI 1993). These scales generated a total score which were recorded for all pair-wise comparisons as short-term follow up data (up to and including six months) or long term follow-up data (greater than six months), or both.

\section{- Adverse events}

\section{Secondary outcomes}

Each of the secondary outcomes were assessed based on a validated assessment scale. The secondary outcomes measured included:

- Change in quality of life, for example, the St George's respiratory questionnaire (SGRQ) (Jones 1991). Generic, validated qualityof-life measures were also considered
- Difference in exercise tolerance, for example, the six-minute walk test (Butland 1982)

- Change in dyspnoea scores, for example, the Borg scale (Borg 1982)

- Change in length of stay or readmission rate

- Change in forced expiratory volume in one second (FEV1)

\section{Timing of outcome assessment}

Time frames were defined as short-term (up to three months), medium-term (three to six months) and long-term follow-up (more than six months). The primary time point used in the 'Summary of findings' table is the longest reported follow-up by each included study. The range of follow-ups for each outcome is described in the 'Summary of findings' table and in the Results section of the review.

\section{Search methods for identification of studies}

\section{Electronic searches}

\section{Cochrane Specialised Registers}

\section{Cochrane Common Mental Disorders Register (CCMDCTR)}

Cochrane Common Mental Disorders maintains a specialised register of RCTs, the CCMDCTR. This register contains over 40,000 reference records (reports of RCTs) for anxiety disorders, depression, bipolar disorder, eating disorders, self-harm and other mental disorders within the scope of this Group. The CCMDCTR is a partially studies-based register with more than $50 \%$ of reference records tagged to c. 12,500 individually PICO-coded study records. Reports of trials for inclusion in the register are collated from (weekly) generic searches of MEDLINE (1950-), Embase (1974-) and PsycINFO (1967-), quarterly searches of the Cochrane Central Register of Controlled Trials (CENTRAL) in the Cochrane Library, and review-specific searches of additional databases. Reports of trials are also sourced from international trials registries, drug companies, the handsearching of key journals, conference proceedings and other (non-Cochrane) systematic reviews and meta-analyses. Details of CCMD's core search strategies (used to identify RCTs) can be found on the Group's website with an example of the core MEDLINE search displayed in Appendix 1.

CCMD's Information Specialist cross-searched the CCMDCTR-Refs and CCMDCTR-Studies registers (to 14 August 2015) using the following terms:

(anxi* or * phobi* or PTSD or post-trauma* or posttrauma or "post trauma*" or "combat disorder" or panic or OCD or obsess" or compulsi* or GAD or stress* or distress ${ }^{\star}$ or neurosis or neuroses or neurotic or psychoneuro*)

AND

((obstruct* and (pulmonary or lung* or airway* or airflow* or bronch $^{\star}$ or respirat $\left.{ }^{\star}\right)$ ) or COPD or emphysema or (chronic ${ }^{\star}$ and bronchiti*))

\section{Cochrane Airways' Register (CAGR)}

Cochrane Airways' Specialised Register is also derived from systematic searches of bibliographic databases including: the Cochrane Central Register of Controlled Trials (CENTRAL; in the Cochrane Library), MEDLINE, Embase, CINAHL, AMED, and PsycINFO, and handsearching of respiratory journals and meeting abstracts (details of the CAGR can be found on the Group's website). 
Cochrane Airways' Information Specialist searched CAGR records coded as 'COPD' for 'Anxiety Disorders' as listed above (14 August 2015).

An additional search of CENTRAL and PsycINFO was conducted at this time, to ensure no records had been missed from these databases in the creation of the CCMDCTR and CAGR (Specialised Registers) (Appendix 2).

Searching the CAGR, CENTRAL and PsycINFO did not retrieve any additional studies beyond those identified by the CCMDCTR, so in 2015 we decided to conduct update searches on the CCMDCTR alone.

\section{National and international trials registers}

Complementary searches were conducted on the World Health Organization International Clinical Trials Registry Platform (ICTRP) and ClinicalTrials.gov.

\section{Searching other resources}

We handsearched reference lists of retrieved, relevant articles to identify any other potentially relevant articles. We contacted authors of potentially-included studies for raw data or unpublished data where required.

\section{Data collection and analysis}

\section{Selection of studies}

Two of three review authors (either KC, ZU or KHM) independently assessed all citations generated from the search strategies to determine whether they satisfied the Criteria for considering studies for this review, through screening of the title, abstract and descriptors. Two of three review authors, as above, retrieved and independently examined the full texts of studies identified as potentially relevant for final inclusion. We resolved disagreements through discussion and by involving a third party if necessary (BS or $A D)$.

\section{Data extraction and management}

Two independent review authors (a combination of ZU, KC and $\mathrm{KHM}$ ) extracted the following data using a standardised and piloted data extraction form, for each included study. The review authors resolved any discrepancies by discussion between themselves and if needed, a third party (BS or AD).

\section{Study eligibility}

Study design, population group and description of psychological therapy.

\section{Participants}

Number of participants, age, gender distribution, ethnicity and comorbidities.

\section{Interventions}

Description of intervention, duration, intensity, who it was delivered by.

\section{Main comparisons}

Comparison 1: Psychological therapies versus no intervention
Comparison 2: Psychological therapies versus education

Comparison 3: Psychological therapies and co-intervention versus co-intervention alone

We stratified these comparisons according to psychological therapy, however, we did not perform an overall pooled estimate for intervention effectiveness for all the psychological therapies in each of these comparisons, that is, we used sub-totals only in the analyses.

\section{Assessment of risk of bias in included studies}

Two review authors independently assessed the risk of bias for all the included studies using the Cochrane tool for assessing risk of bias, which is a domain-based evaluation (Higgins 2011a). We assessed risk of bias as 'Low risk of bias', 'High risk of bias' and 'Unclear risk of bias' as per the guidelines in the Cochrane Handbook for Systematic Reviews of Interventions, table 8.5.c (Higgins 2011a). We resolved any conflicts in the assessment either by consensus or by referring to a third party. The domains we evaluated were:

\section{Sequence generation}

Methods considered adequate included: random number table, computer random-number generator, coin toss, shuffling cards or envelopes, throwing dice and drawing lots.

\section{Allocation concealment}

Methods considered adequate included: central allocation (phone, web, pharmacy), sequentially-numbered identical drug containers and serially-numbered sealed and opaque envelopes.

\section{Blinding (of participants)}

We considered blinding adequate if: trial authors mentioned that participants were blinded to the intervention, although for psychological therapies this was unlikely due to the difficulties associated with delivery of communication-based interventions.

\section{Blinding (of outcome assessors)}

We considered blinding adequate if: authors mentioned that outcome assessors were blinded to sequence allocation.

\section{Incomplete outcome data}

We assessed risk of bias due to incomplete outcome data on the grounds of whether the incomplete outcome data were adequately addressed or not, as per the Cochrane Handbook for Systematic Reviews of Interventions section 8.12.

\section{Selective outcome reporting}

We considered studies to be at low risk of bias if a protocol was available and all prespecified outcomes were reported in the prespecified way, or in the absence of a protocol, if all expected outcomes were reported (and as per recommendations in the Cochrane Handbook for Systematic Reviews of Interventions, table 8.5.c.

\section{Other bias}

We considered studies at a low risk of other bias if they were conducted in such a way as to ensure no other influencing factors that could potentially affect the outcome were evident. 
Examples of other biases included: extreme baseline imbalances for participants or outcomes, contamination of the intervention or control group, and selective recruitment of study participants.

We have presented the results of the 'Risk of bias' assessment in a 'Risk of bias' table and described them narratively within the results of the review.

\section{Measures of treatment effect}

\section{Continuous data}

We summarised available data by either mean differences (MD) or standardised mean differences (SMD) where appropriate, using mean values and standard deviations. We consulted a statistician for additional support where required (AE) (Deeks 2011).

\section{Dichotomous date}

Had dichotomous data been presented we would have calculated odds ratios with $95 \%$ confidence intervals. We would have presented data as either final values (post-intervention) or as change from baseline, if we had not been able to retrieve raw data from the trialists (Deeks 2011).

\section{Unit of analysis issues}

\section{Cluster-randomised trials}

Cluster-randomised controlled trials, that is, trials in which outcomes relate to individual participants, whilst allocation to the intervention is by hospital, clinic or practitioner, may introduce unit of analysis errors. Using statistical methods that assume, for example, that all participants' chances of benefit are independent, ignores the possible similarity between outcomes for participants seen by the same provider. This may underestimate standard errors and give misleadingly narrow confidence intervals, leading to the possibility of a type 1 error (Altman 1997). For cluster-randomised studies, we performed analyses at the level of individuals, whilst accounting for the clustering in the data using a random-effects model for pooled meta-analysis, as recommended in the Cochrane Handbook for Systematic Reviews of Interventions (chapter 16.3.3) (Higgins 2011b) (checked by a statistician (AE)). For those studies that did not adjust for clustering, we replaced the actual sample size with the effective sample size (ESS), calculated using a rho $=0.02$ (as per Campbell 2000).

\section{Cross-over trials}

We extracted data from cross-over studies for the first phase only (pre-cross-over), due to the potential for a significant carry-over effect for psychological therapies.

\section{Studies with multiple treatment groups}

We considered multi-arm trials for inclusions provided that there was an intervention arm with any of the interventions mentioned in the experimental group above and a control arm with any of the comparators mentioned above. In the case of multi-arm trials we included each pair-wise comparison separately, but with shared intervention groups divided out approximately evenly among the comparators. However, in cases where the intervention groups were deemed similar enough to be pooled, we combined the groups using appropriate formulae in the Cochrane Handbook for Systematic Reviews of Interventions (table 7.7.a for continuous data
(Higgins 2011c) and chapter 16.5.4 for dichotomous data, (Higgins 2011b)).

\section{Dealing with missing data}

We evaluated missing information regarding participants on an available case-analysis basis as described in chapter 16.2.2 of the Cochrane Handbook for Systematic Reviews of Interventions (Higgins 2011b). Had there been statistics essential for analysis missing (e.g. group means and standard deviations (SDs) for both groups not reported) that could not be calculated from other data, we would have attempted to contact the study authors to obtain the data. Any loss of participants that occurred before the baseline measurements were performed would not have affected the eventual outcome data of the study. Any losses after the baseline measurements were taken may have affected trial validity. For dropouts at the initial phase of a trial (by the end of the second week of intervention/placebo administration), we did not include their data and for these studies we used the final data from the completers only. For participants who dropped out after the second week or with unclear dropout time, we used the last observation carried forward (LOCF) as presented in the publications, or had the data been missing we would have obtained raw data. Had we been unsuccessful in obtaining this raw data, we would have reported the missing data under 'other' sources of bias in the 'Risk of bias' tables and discussed the details in the text.

\section{Assessment of heterogeneity}

We expected this review to have some heterogeneity, with factors such as baseline severity of anxiety, severity of underlying COPD or consistency of diagnostic thresholds for COPD, or both, time of measurement of results and varying measuring tools used to assess outcomes contributing. We used the $\mathrm{Chi}^{2}$ (Deeks 2011) and 12 statistics (Higgins 2003) to quantify inconsistency across studies in combination with visual inspection of the data for differences between studies (e.g. types of interventions, participants etc.). Thresholds for the interpretation of the 12 statistic can be misleading, since the importance of inconsistency depends on several factors (Deeks 2011). These include magnitude and direction of the effect and strength of the evidence for heterogeneity, for example the $\mathrm{P}$ value from the $\mathrm{Chi}^{2}$ test, or a confidence interval for $\mathrm{1}^{2}$. For the purpose of this review, we considered an 12 statistic representing substantial or considerable heterogeneity for further investigation through subgroup analyses to examine possible causes, as per chapter 9.5.2 of the Cochrane Handbook for Systematic Reviews of Interventions (Deeks 2011). The overlapping bands for the $\mathrm{I}^{2}$ statistic are:

- $0 \%$ to $40 \%$ : might not be important;

- $30 \%$ to $60 \%$ : may represent moderate heterogeneity;

- $50 \%$ to $90 \%$ : may represent substantial heterogeneity;

- $75 \%$ to $100 \%$ : considerable heterogeneity.

\section{Assessment of reporting biases}

Since there were fewer than 10 studies, where we identified reporting biases we extrapolated them within the 'other bias' section in the 'Risk of bias' tables. If in future versions of this review we include more than 10 studies in any analysis, we will assess potential reporting biases using a funnel plot. Asymmetry in the plot might be attributed to publication bias, but may well be due to true heterogeneity or a poor methodological design. In 
case of asymmetry, contour lines may be included corresponding to perceived milestones of statistical significance $(P=0.01,0.05$, 0.1 etc.) to funnel plots, which may help to differentiate between asymmetry due to publication bias from that due to other factors (Sterne 2011).

\section{Data synthesis}

We pooled the extracted data in meta-analyses using the randomeffects model to allow for expected heterogeneity (due to expected differences in the interventions and populations). We assessed all the included studies for inclusion in the primary analyses, and performed a sensitivity analysis for studies which were at an unclear or high risk of bias for sequence generation and allocation concealment, and for studies involving participants who had significant co-morbidities, for example, dementia or severe heart failure. We performed separate meta-analyses for intervention subgroups as defined under Subgroup analysis and investigation of heterogeneity (Deeks 2011). For trials reporting data at more than one point in time, we extracted data from the final follow-up period reported by trialists. We analysed data using Review Manager 5 (RevMan 5) software (RevMan 2014).

\section{Subgroup analysis and investigation of heterogeneity}

We have prespecified subgroups to investigate this heterogeneity to reduce the likelihood of spurious findings, first by limiting the number of subgroups investigated and second by preventing knowledge of the studies' results influencing which subgroups are analysed (Deeks 2011). These contributing factors were identified as relevant in our previous completed review for pharmacological interventions for anxiety in COPD (Usmani 2011). We have described all included studies in table and narrative form reporting on study design, population, intervention characteristics and outcome measures.

We performed subgroup analyses for the above-mentioned psychological therapies according to:

- duration of intervention (e.g. 0 to 3 months, 3 to 6 months, $>6$ months); and

- severity of anxiety symptoms (i.e. mild, moderate and severe accepting the study authors' definition of this).

These subgroups permit an examination into the possible causes of heterogeneity, however their primary objective is to extrapolate data for the purposes of forming hypotheses.

\section{Sensitivity analysis}

Had there been sufficient data we would have conducted a sensitivity analysis to examine the effects of methodological decisions taken throughout the review process, particularly in regard to the inclusion criteria (Deeks 2011). We would have tested the validity and robustness of the findings by removing studies based on the following criteria:

- inadequate sequence generation;

- inadequate allocation concealment;

- significant attrition of the study population (20\% or higher attrition);

- studies on populations with significant co-morbidities;

- cluster-randomised trials;

- cross-over studies;

- studies containing data imputed by the review authors.

\section{'Summary of findings' table}

As there were only three included studies in this review, the 'Summary of findings' table was limited only to the data reported under comparison 3 (psychological interventions and cointerventions versus co-interventions alone). Follow-up periods presented in the 'Summary of findings' table relate to the final follow-up reported in each study for every outcome measure (range of follow-up is specified in the 'Summary of findings' table). We used only published data to populate information in the 'Summary of findings' table. We used the GRADE approach to assess the quality of evidence (GRADE 2013). The source of all information used in the table is from the publications only. The first four outcomes listed above under Types of outcome measures are the same outcomes used in the 'Summary of findings' table (Schünemann 2011).

\section{RES U LTS}

\section{Description of studies}

\section{Results of the search}

We identified a total of 675 records from searching Cochrane specialised registers and bibliographic databases (to 14th of Aug 2015) with 579 short-listed once duplicates were removed. We retrieved an additional 72 records from screening clinical trials registries and identified one citation via author contact. This resulted in a total of 647 records screened and 32 full-text articles (including online protocols) identified as potentially eligible. From these, we included three studies (five full-text articles) in the final qualitative and quantitative analyses. Seven studies are awaiting classification and two were ongoing at the time of review completion (Figure 1). 
Figure 1. Study flow diagram

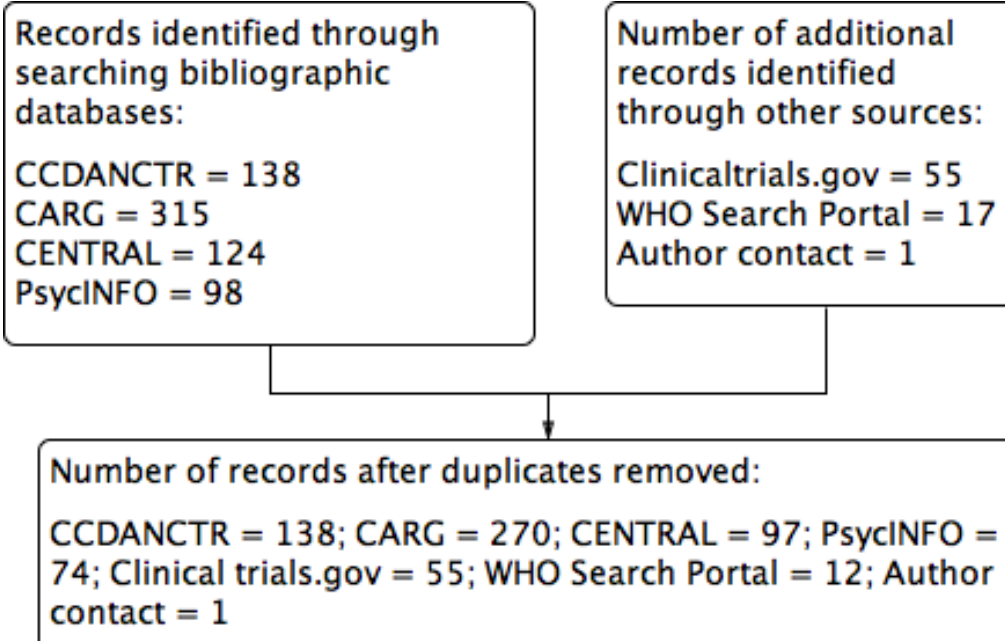

Number of records after duplicates removed:

CCDANCTR $=138 ;$ CARG $=270 ;$ CENTRAL $=97 ;$ PsycINFO $=$ 74; Clinical trials.gov $=55 ;$ WHO Search Portal $=12$; Author contact $=1$

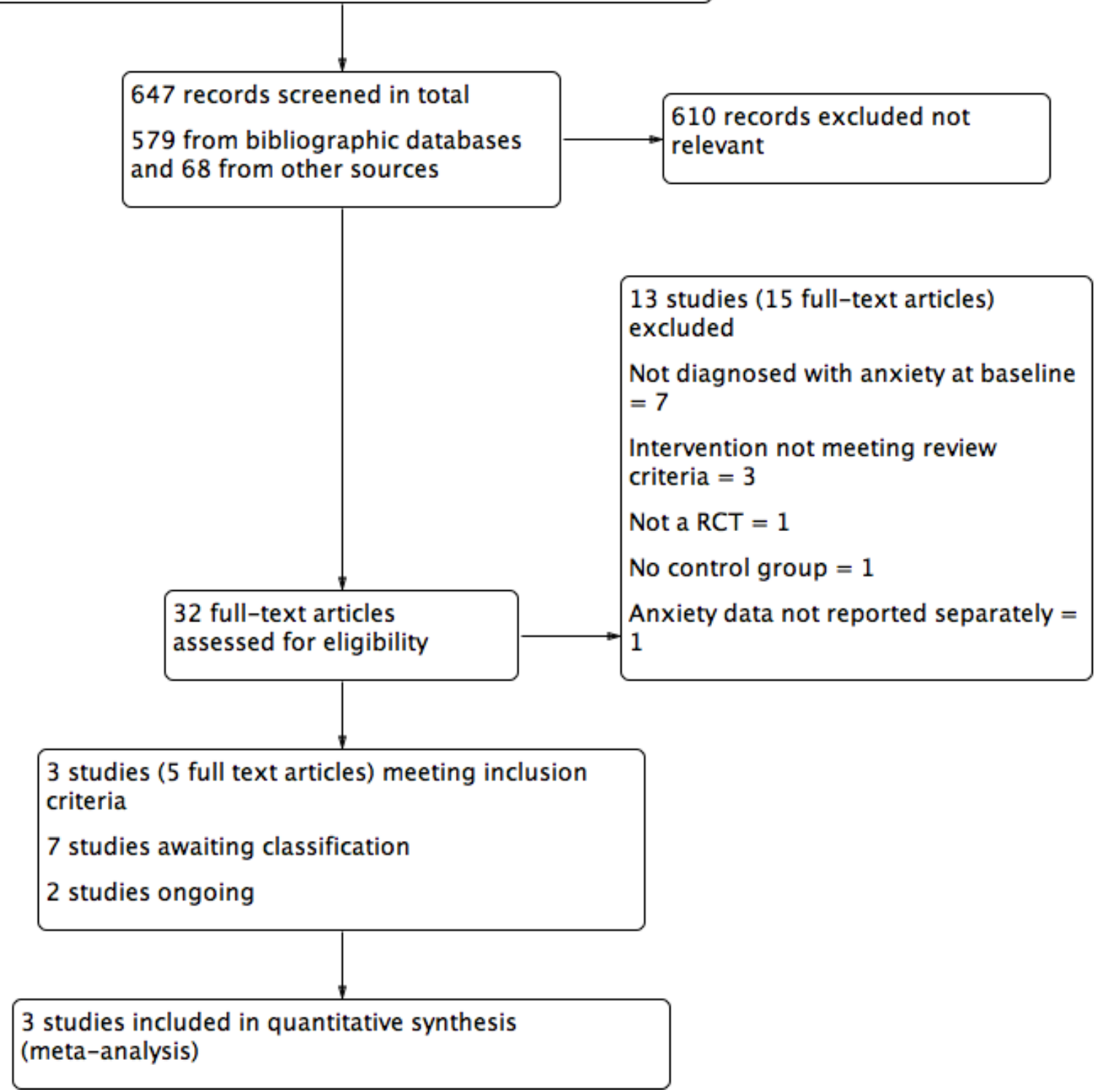




\section{Included studies}

We included three studies in this review, with the following characteristics (see also Characteristics of included studies).

\section{Design}

All three studies were single-centre, parallel RCTs coming from Brazil (de Godoy 2003), Norway (Hynninen 2010) and the USA (Kunik 2008).

\section{Sample sizes}

Each study reported difficulties with sample size, two being unable to meet the target recruitment number (Kunik 2008; Hynninen 2010) and the third (de Godoy 2003) not reporting a prespecified sample size calculation. Samples sizes ranged from $n=30$ in de Godoy 2003, n = 51 in Hynninen 2010 and the largest being $n=238$ in Kunik 2008.

\section{Setting}

Specific settings for psychological therapies were not described in detail, however two studies did report group settings for intervention delivery with an average of five participants in each session for the Hynninen 2010 study and up to 10 participants in each session for the Kunik 2008 study, whilst the setting for de Godoy 2003 was unclear. Participants of the Kunik 2008 study were recruited from a Veterans Affairs hospital, whilst the Hynninen 2010 study used outpatients from a University hospital and de Godoy 2003 recruited from a pulmonary rehabilitation clinic.

\section{Participants}

All three studies recruited people with diagnosed COPD and anxiety using a validated tool. Although all studies recruited participants from the hospital setting, two studies (Kunik 2008; Hynninen 2010) also used external advertisements/flyers to attract participants into the study. The male to female ratio in the Hynninen 2010 study was similar, however there were more men recruited into both the de Godoy 2003 study (22 men and 8 women) and the Kunik 2008 study (226 men and 9 women). Only the Kunik 2008 study reported on participant ethnicity with 192 white, seven Hispanic and 38 black participants recruited. Of the 256 eligible participants for the Kunik 2008 study, 238 were randomised but only 181 attended their first session ( $24 \%$ attrition before the intervention commenced).

\section{Interventions and comparators}

de Godoy 2003 used psychotherapy in addition to exercise, physiotherapy and education in comparison to exercise, physiotherapy and education alone. Hynninen 2010 and Kunik 2008 both employed Cognitive and Behavioural Therapy (CBT) in addition to co-interventions of telephone counselling and group discussions for each study respectively. The control population of the Hynninen 2010 study used an enhanced standard care programme for COPD with regular telephone contacts whilst the Kunik 2008 control group received COPD education. Although we defined the control of the Kunik 2008 study to be a cointervention, it could also have been considered as an educationonly intervention. However, due to the small number of included studies in this review (three studies) we classified all studies as co-interventions, which also facilitated meta-analysis and interpretation of results.

\section{Outcomes}

All three studies measured anxiety using the Beck Anxiety Inventory (BAI). A number of secondary outcomes were also assessed including depression, quality of life, exercise capacity and service utilisation.

\section{Excluded studies}

We excluded 13 of the potentially eligible studies (15 full-text articles) from the review due to no diagnosis of anxiety at baseline ( $\mathrm{n}$ = 7; Aubuchon 1990; Blake 1990; Gift 1992; Livermore 2010a; Williams 2011; Doyle 2013; Blumenthal 2014), the intervention not meeting the criteria for inclusion as defined in the protocol $(n=3)$ due to being either an intervention of progressive muscle relaxation without a psychological intervention (Lolak 2008), or due to being a multi-component intervention (Pommer 2012; Yang 2015), and we excluded the remaining three studies because they were not randomised (Cully 2007), did not have a control group (de Godoy 2005) or did not report anxiety data separately (Lamers 2010). See Characteristics of excluded studies.

\section{Ongoing studies}

We identified seven studies as ongoing at the time of review completion. See Characteristics of ongoing studies for individual trial details. We identified all the studies through either published protocols in manuscript format or via online clinical trials registries. It is unclear if all inclusion/exclusion criteria as outlined in the protocol of this review will be met for each study due to the limitations in reported data. One study is reported to use mindfulness-based cognitive therapy (Farver-Vestergaard 2014), whilst the remaining six studies specifically report the use of CBT.

\section{Studies awaiting classification}

We classified two studies as 'awaiting classification'. One study was a Chinese paper that could not be translated in time for inclusion in this review (Shao 2003). Fifty-four people with COPD were randomised to a behavioural therapy with psychological and somatic components or usual care, however, it is unclear if the presence of baseline anxiety was an inclusion criteria or if people with COPD were formally diagnosed as per the requirements for inclusion within this review (see Characteristics of studies awaiting classification). For the other study (Livermore 2015) there is a question surrounding baseline anxiety scores for individual participants to determine eligibility of subjects for this review. We will continue to attempt to contact the study authors and, if no response has been received by the time of the next update, this study will be moved to the excluded category.

\section{Risk of bias in included studies}

For details of the risk of bias judgements for each study, see Characteristics of included studies. We have presented a graphical representation of the overall risk of bias in included studies in Figure 2 and Figure 3. 
Figure 2. Risk of bias graph: review authors' judgements about each risk of bias item presented as percentages across all included studies

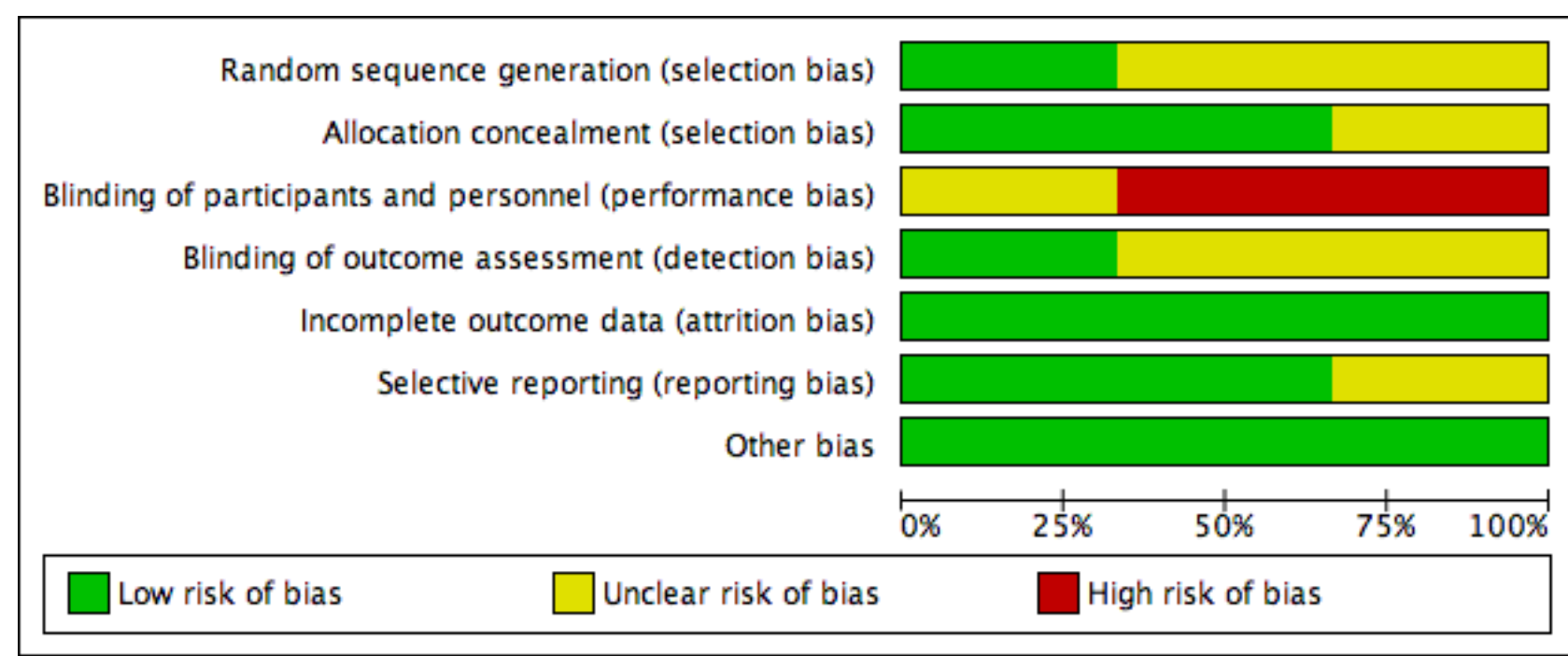


Figure 3. Risk of bias summary: review authors' judgements about each risk of bias item for each included study

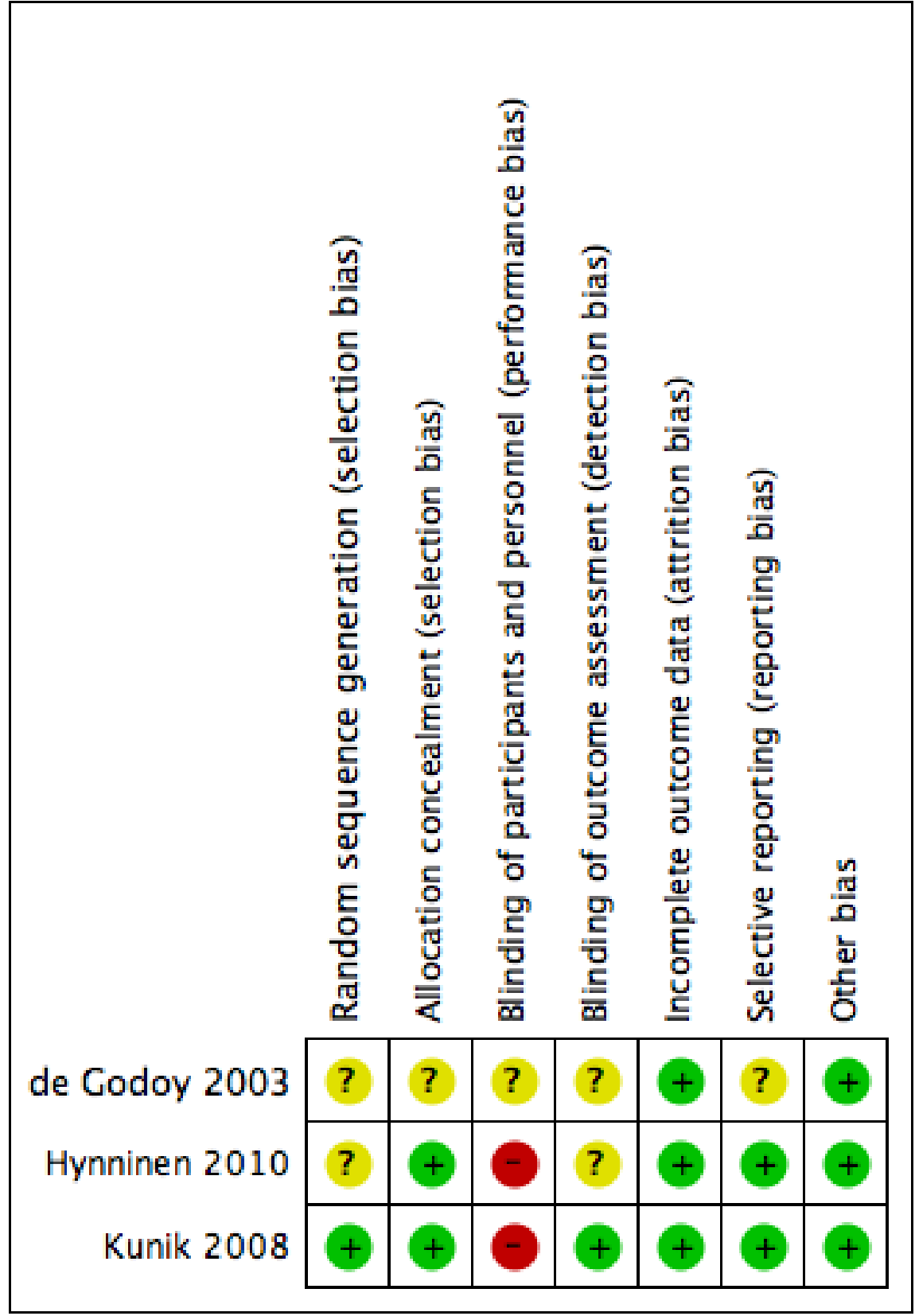

\section{Sequence generation (selection bias)}

Only one study adequately reported on method of sequence generation (Kunik 2008) using a computer program with blocks to provide appropriately equal numbers per class of COPD. The instructor assigned treatment to the code initially through flipping a coin. The two remaining studies reported randomisation as occurring, however, they did not describe their methods in detail.

\section{Allocation}

Two studies sufficiently reported allocation concealment using an external statistician to provide treatment codes to the study coordinator once sufficient numbers of participants for two classes had been recruited (Kunik 2008) and by using numbered containers that were identical in appearance for the two groups (Hynninen
2010). The de Godoy 2003 study did not report any details of allocation concealment.

\section{Blinding}

Blinding of participants and personnel (performance bias)

Blinding to psychological therapies can be challenging unless an active comparator arm, for example, education or sham interventions is included. Two studies did not blind participants or personnel to the treatment allocation, whilst the third study (de Godoy 2003) reported that group 1 was blinded in relation to the activities of group 2 and vice versa. 


\section{Blinding of outcome assessors (detection bias)}

Outcome assessor blinding was unclear in two studies and assessed to be a low risk of bias in the Kunik 2008 study as authors reported that study personnel performing assessments were blinded to treatment condition.

\section{Incomplete outcome data}

All three studies had a low risk of bias for incomplete outcome data as they used intention-to-treat analyses. Moreover, any missing data were reported to be accounted for in the analyses.

\section{Selective reporting}

Selective reporting was a low risk in two studies due to the availability of prespecified published protocols, allowing adequate comparison of prespecified outcomes and those reported in the publications. We assessed the de Godoy 2003 study as having an unclear risk of bias due to the inability to review a prespecified protocol.

\section{Other potential sources of bias}

Had there been a sufficient number of studies to properly assess reporting bias, we would have assessed potential reporting biases using a funnel plot. Asymmetry in the plot may have been attributed to publication bias, but may well be due to true heterogeneity or a poor methodological design. In case of asymmetry, contour lines would have been included corresponding to perceived milestones of statistical significance $(P=0.01,0.05$, 0.1 etc.) to funnel plots, which may have helped to differentiate between asymmetry due to publication bias from that due to other factors (Higgins 2011a). No additional formal testing for funnel plot asymmetry would have been performed.

Although we defined the control of the Kunik 2008 study to be a cointervention, it could also have been considered as an education- only intervention. However, due to the small number of included studies in this review (three studies) we pooled all studies together to facilitate meta-analysis.

\section{Effects of interventions}

See: Summary of findings for the main comparison Psychological therapies for anxiety for people with COPD

\section{Comparison 1: Psychological therapies versus no intervention}

There were no data for this comparison.

\section{Comparison 2: Psychological therapies versus education}

There were no data for this comparison.

\section{Comparison 3: Psychological therapies and co-intervention versus co-intervention alone}

Three studies including 319 participants contributed data to this comparison. See also: Summary of findings for the main comparison.

\section{Primary outcomes}

\section{Change in anxiety symptoms}

Psychological therapies were observed to be more effective for the treatment of anxiety compared with co-interventions or usual care (mean difference (MD) $-4.41,95 \%$ confidence interval (CI) -8.28 to -0.53 ; participants $=319 ; \mathrm{I}^{2}=62 \% ; \mathrm{P}=0.03$; Analysis 1.1; Figure 4). However, there was substantial heterogeneity $(12=62 \%)$, which limits the reliability of the findings. In the study by de Godoy 2003 the psychotherapy group started with a BAl score of $12.9 \pm 6.9$, whilst the control population was $10.9 \pm 9.8$. Authors reported a change from baseline in the intervention arm $(P<0.001)$, though no change was observed from baseline to follow-up in the control group $(9.2 \pm 8.6 ; \mathrm{P}=0.15)$.

Figure 4. Forest plot of comparison 1: Psychological therapies versus co-intervention, outcome: 1.1 Anxiety

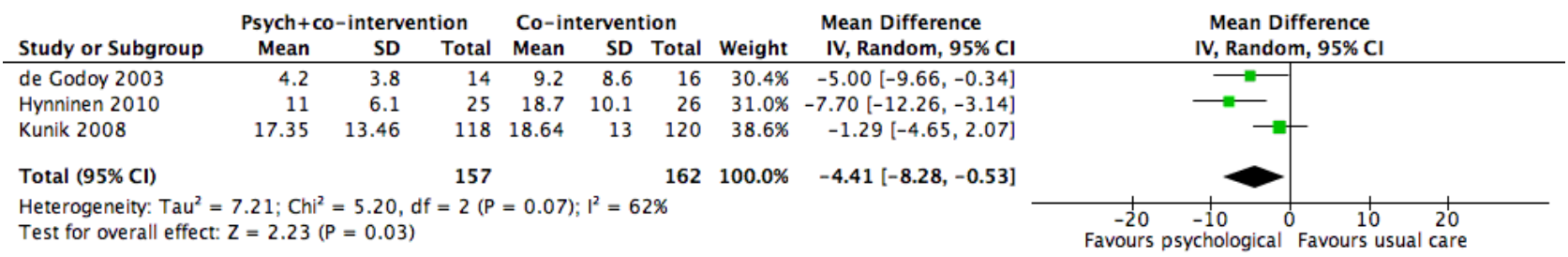

\section{Adverse events}

No adverse events were reported for any of the included studies.

\section{Secondary outcomes}

\section{Change in quality of life}

Two studies with 289 participants reported quality of life through use of three different tools, being SGRQ, SF36 and CRQ. Psychological interventions were observed to be more effective compared to the control population for the combination of SGRQ and the physical composite of SF36 (standardised mean difference (SMD) $-0.40,95 \% \mathrm{Cl}-0.88$ to -0.08 ; participants $=289$; studies $=2$; $\left.\mathrm{I}^{2}=61 \% ; \mathrm{P}=0.10\right)$. No evidence of any effect was observed for SGRQ and the emotional composite of SF36 (SMD $-0.30,95 \% \mathrm{Cl}$ -1.03 to 0.44 ; participants $=289$; studies $=2 ; 12=82 \%$ ); Analysis 1.2 ).
Of note substantial heterogeneity was observed in these analyses. Alongside SF36, CRQ was also reported in the Kunik 2008 study with only one of the eight categories producing results in favour of the CBT group for general health (end of treatment eight-week score: intervention arm mean $34.14 \pm 28.53$ and control arm mean 37.76 $\pm 27.74 ; \mathrm{P}=0.05$ ).

\section{Difference in exercise tolerance}

Compared with the control, there was no significant difference in the number of steps taken as measured by the Six Minute Walking Distance (6MWD) when engaged in psychological therapies: (MD $-2.78,95 \% \mathrm{Cl}-58.49$ to 52.94 , participants $=268$; studies $=2 ; \mathrm{I}^{2}=$ $80 \%$; Analysis 1.3). The Kunik 2008 study, which was included in the meta-analysis, examined 6MWD at eight weeks' (post-intervention) and again at 12 months' follow-up with a difference in favour of the 
control arm at 12 months $(P=0.05)$. However, the study authors reported that group means at the beginning of the follow-up period were not equal $(P<0.01)$, contributing to the spurious finding.

Exercise capacity in the psychotherapy group of the de Godoy 2003 study improved almost twice as much from baseline as the control population $(P=0.11)$. The study authors reported that it may have been the improvement in physical functioning that facilitated improvement in the psychological variables, rather than the psychotherapy itself. ).

\section{Change in dyspnoea scores}

Only one study (Kunik 2008) reported dyspnoea via CRQ, with an improvement in the CBT group over that of control at eight-week end-of-treatment follow-up (intervention group mean $3.36 \pm 1.64$; control group mean $3.51+1.59 ; \mathrm{P}=0.02$ ).

\section{Change in length of stay or readmission rate}

No studies provided data specifically on length of stay or hospital readmission rates. However the Kunik 2008 study did indirectly examine this by tracking the number of treatment visits pre and post study. No differences were observed for the ratio of pre treatment to post treatment between groups for outpatient visits, hospital admissions, mental health visits and emergency visits per month, per participant.

\section{Change in forced expiratory volume in one second (FEV1)}

No studies reported on FEV1. Whilst FEV1 is helpful to note the severity of COPD, as with most interventions few if any other therapies, including pharmacotherapies significantly improve FEV1 in COPD.

\section{Subgroup analyses}

We carried out subgroup analyses for duration of follow-up due to multiple follow-up periods reported in the Kunik 2008 study (eight weeks, i.e. post treatment, and 12 months) and the Hynninen 2010 study (seven weeks and six months). In both anxiety and quality-oflife outcomes, results became better (favoured intervention more) with longer follow-up compared to immediately post treatment. In the case of anxiety, short-term ( 0 to 3 month follow-up) benefits were seen in favour of the intervention arm with a MD of $-3.86 ; 95 \%$ $\mathrm{Cl}$ of -6.63 to -1.10 and $\mathrm{P}=0.006\left(\mathrm{I}^{2}=19 \%\right)$, whilst these findings were not maintained long-term ( 6 to 12 months; MD -4.30 with $95 \%$ $\mathrm{Cl}$ of -10.57 to 1.97 and $\mathrm{P}=0.18 ; \mathrm{I}^{2}=80 \%$; Analysis 2.1 ). We did not note any evidence of an effect for exercise capacity (Analysis 2.2) or quality of life (Analysis 2.3, Analysis 2.4), even after sub group analyses.

\section{DISCUSSION}

\section{Summary of main results}

Based on the small number of included studies within this review (only three), it is difficult to draw any meaningful and reliable conclusions. Some evidence of improvement in anxiety was observed with a benefit in favour of the psychological therapy arm, however, the presence of substantial heterogeneity and low quality of the evidence limits the reliability of these findings. Six-minute walking distance was analysed in two of the three included studies, producing no evidence of any effect. As such there is insufficient information to draw reliable conclusions about whether psychological therapies are beneficial for people with COPD with anxiety (see Summary of findings for the main comparison).

Of note however, sub-group analyses for duration of follow-up for both anxiety and quality of life indicate the potential for greater benefits in favour of the psychological intervention given more time (in the presence of wider confidence intervals due to small number of relevant studies). Although intervention treatment lasted for similar periods of time (eight weeks and seven weeks for the Kunik 2008 and Hynninen 2010 studies respectively), participants within both studies did better at long-term follow-up in comparison to immediately post-intervention. This suggests that psychological interventions may take longer to work than during the treatment phase alone.

\section{Overall completeness and applicability of evidence}

A limitation of this review is that all three included studies recruited participants with both anxiety and depression, not just anxiety, which may confound the results, as presence of co-morbid depression may have over estimated or under estimated the effect of intervention in different participants (based on different levels of severity of anxiety and depression), the details of which are out of the scope of this review. Also important to note is that the majority of the participants of this review were men. From the prespecified outcomes identified as being important for this review, none of the three included studies included as an outcome either adverse events or objective markers of lung disease, such as FEV1. Length of stay and readmission rate was also not effectively evaluated in any of the three studies. Considering the small number of included studies and limited reporting for each of the outcomes, the overall completeness and applicability of evidence is poor. As such, findings from this review should be interpreted with caution and the questions posed in this review remain unanswered.

\section{Quality of the evidence}

The quality of evidence in this review overall was low, with two of the three studies having prespecified protocols available for comparison to methods, and outcomes reported on within the final publications. Although all studies reported that randomisation occurred, only one provided a description of the methods for sequence generation. Allocation concealment was adequately reported in two studies whilst the third was unclear.

\section{Consistency}

Substantial heterogeneity was identified in all meta-analyses across studies, suggesting the possibility of these being true differences in the underlying treatment effect. However, as we only included three studies, it is difficult effectively to examine consistency. As such, the quality of results was downgraded due to inconsistency.

\section{Indirectness}

Due to the small number of included studies and broad classification of psychological therapies, it is likely that this review contains indirectness through varying interventions, comparators, populations and even outcomes and the use of different reporting tools. 


\section{Imprecision}

Imprecision was identified particularly when examining exercise capacity with the presence of wide confidence intervals around the treatment effect. This is of particular concern when there are few studies with few participants attempting to determine the results of a particular outcome. As such the quality of the 6MWD outcome was downgraded due to imprecision.

\section{Publication bias}

It is difficult to determine the presence of publication bias and systematic underestimation or overestimation of the underlying beneficial or harmful effect due to inclusion of only three studies. Screening of online clinical trial registries allows identification of studies regardless of a positive or negative effect for the treatment, which may reduce the possibility of publication bias. The identification of six ongoing studies and one awaiting classification is reassuring, however, there is the possibility of negative studies being missed before publication of protocols became mandatory.

\section{Potential biases in the review process}

As the three studies identified for inclusion within the review were from three different countries and different healthcare settings, it is difficult to determine the extent to which the results can be generalised. In particular, all three studies reported issues with either prespecified sample sizes not being met (Kunik 2008; Hynninen 2010) or a small sample limiting the ability to draw reliable conclusions from the results (de Godoy 2003, in which there was no mention of sample size calculations). Authors in the Hynninen 2010 trial reported that, due to the low sample size of their study, it was possible that significant differences had not been detected when they existed. They also reported that it may have been possible to detect a difference on anxiety symptoms due to age if a larger sample size had been achieved. Low recruitment numbers in the Kunik 2008 study was reported to be due to difficulties often occurring with recruitment of Veteran Affairs' patients due to greater prevalence of physical and mental health problems. Authors of the de Godoy 2003 study suggested that, due to the relatively small sample size, the conclusions from their study should only be considered as tentative.

An additional limitation relates to risk of bias categories deemed to be unclear by review authors, as we did not contact individual study authors for clarification or to obtain further information.

\section{Agreements and disagreements with other studies or reviews}

Meta-analyses attempting to examine the impact of psychological interventions for anxiety in people with COPD have consistently identified a lack of methodologically rigorous evidence to support treatment efficacy (Rose 2002; Mikkelsen 2004; Baraniak 2011), resulting in the inability to draw reliable conclusions. A 2014 overview examining the prevalence, impact and management of depression and anxiety in COPD also found that the quality of evidence underpinning treatment varied considerably (Panagioti 2014). The authors found that interventions that included pulmonary rehabilitation with or without psychological components improved symptoms of depression and anxiety in COPD. They considered cognitive and behavioural therapy and, though treatment effects were small, they concluded that this type of therapy could potentially lead to greater benefits in anxiety for people with COPD if embedded in multi-disciplinary collaborative frameworks. Another systematic review and metaanalysis of complex interventions for depression and anxiety in COPD identified 28 studies for inclusion (Coventry 2013). Subgroup analyses identified small but non-significant effects on self-reported anxiety symptoms post-treatment for cognitive and behavioural interventions (SMD $-0.17 ; 95 \% \mathrm{Cl}-0.35$ to 0.01 ; 7 studies) and relaxation (SMD $-0.18 ; 95 \% \mathrm{Cl}-0.67$ to 0.30 ; 3 studies), but observed no evidence of any effect for selfmanagement education (SMD $-0.00 ; 95 \% \mathrm{Cl}-0.17$ to $0.16 ; 5$ studies). This review observed an improvement with the intervention for multi-component exercise training, though the sample included substantial heterogeneity (SMD $-0.47 ; 95 \% \mathrm{Cl}-0.66$ to $-0.28 ; \mathrm{P}=$ 0.002; 14 studies; $I^{2}=63.3 \%$; Coventry 2013 ).

\section{AUTHORS' CONCLUSIONS}

\section{Implications for practice}

Based on the small number of included studies identified for inclusion within this review (only three), it is difficult to draw any meaningful and reliable conclusions. All three included studies recruited participants with both anxiety and depression, not just anxiety (which may confound the results) and compared psychotherapy with co-intervention versus co-intervention alone. Some evidence of improvement in anxiety was observed in the psychological therapy arm, however, the low quality of the evidence and presence of substantial heterogeneity limits our ability to draw any conclusions from these findings.

\section{Implications for research}

There is a need for further research trials to evaluate the role of psychotherapy for anxiety in people with chronic obstructive pulmonary disease (COPD) using a population with anxiety symptoms and COPD. Given problems with sample size, participant selection and only one type of psychotherapy in current studies, future researchers might need to consider multi-centre studies with different modalities of psychotherapy other than cognitive behavioural therapy. New research should also assess adverse events, the effect on hospital admissions, length of stay and longterm effects on quality of life produced by psychotherapy.

\section{ACK N O WLEDGEMENTS}

We acknowledge the assistance of the editors and staff of the Cochrane Common Mental Disorders Group.

\section{CRG Funding Acknowledgement}

The National Institute for Health Research (NIHR) is the largest single funder of Cochrane Common Mental Disorders.

\section{Disclaimer}

The views and opinions expressed therein are those of the review authors and do not necessarily reflect those of the NIHR, NHS or the Department of Health. 


\section{R E F E R E N C E S}

\section{References to studies included in this review}

\section{de Godoy 2003 \{published data only\}}

de Godoy DV, de Godoy RF. A randomised controlled trial of the effect of psychotherapy on anxiety and depression in chronic obstructive pulmonary disease. Archives of Physical Medicine and Rehabilitation 2003;84(8):1154-7.

\section{Hynninen 2010 \{published data only\}}

* Hynninen MJ, Bjerke N, Pallesen S, Bakke PS, Nordhus IH. A randomised controlled trial of cognitive behavioral therapy for anxiety and depression in COPD. Respiratory Medicine 2010;104:986-94.

Nordhus IH. Cognitive Behavioral Therapy for Anxiety and Depression in COPD. clinicaltrials.gov/ct2/show/NCT00545922 2007.

\section{Kunik 2008 \{published data only\}}

Kunik ME, DeBakey ME. A Cognitive-Behavioral Intervention for Depression and Anxiety in COPD. clinicaltrials.gov/ct2/show/ NCT00105911 2005.

* Kunik ME, Veazey C, Cully JA, Souchek J, Graham DP, Hopko D, et al. COPD education and cognitive behavioral therapy group treatment for clinically significant symptoms of depression and anxiety in COPD patients: a randomised controlled trial. Psychological Medicine 2008;38:385-96.

\section{References to studies excluded from this review}

\section{Aubuchon 1990 \{published data only\}}

Aubuchon BL. The effects of positive mental imagery on hope, coping, anxiety, dyspnea and pulmonary function in persons with chronic obstructive pulmonary disease: tests of a nursing intervention and a theoretical model [Dissertation]. Austin (USA): The University of Texas at Austin, 1990.

Blake 1990 \{published data only\}

Blake RL Jr, Vandiver TA, Braun S, Bertuso DD, Straub V. A randomised controlled evaluation of a psychosocial intervention in adults with chronic lung disease. Family Medicine 1990;22(5):365-70.

\section{Blumenthal 2014 \{published data only\}}

Blumenthal JA, Emery CF, Smith PJ, Keefe FJ, WeltyWolf $\mathrm{K}, \mathrm{Mabe} \mathrm{S}$, et al. The effects of a telehealth coping skills intervention on outcomes in chronic obstructive pulmonary disease: primary results from the INSPIRE-II study. Psychosomatic Medicine 2014;76:581-92.

\section{Cully 2007 \{published data only\}}

Cully J. Transforming Psychotherapy for Chronically III Patients. clinicaltrials.gov/ct2/show/NCT00520429 2007.

\section{de Godoy 2005 \{published data only\}}

de Godoy DV, de Godoy RF, Junior BB, Vaccari PF, Michelli M, Teixeira PJZ. The effect of psychotherapy provided as part of a pulmonary rehabilitation program for the treatment of patients with chronic obstructive pulmonary disease. Jornal Brasileiro de Pneumologia 2005;31(6):499-505.

Doyle 2013 \{published data only\}

Doyle T, Palmer S, Johnson J, Babyak M, Smith P, Mabe S. Association of anxiety and depression with pulmonaryspecific symptoms in chronic obstructive pulmonary disease. International Journal of Psychiatry in Medicine 2013;45(2):189-202.

Gift 1992 \{published data only\}

Gift AG, Moore T, Soeken K. Relaxation to reduce dyspnea and anxiety in COPD patients. Nursing Research 1992;41(4):242-6.

\section{Lamers 2010 \{published data only\}}

Lamers F, Jonkers CCM, Bosma H, Chavannes NH, Knottnerus JA, Van Eijk JT. Improving quality of life in depressed COPD patients: Effectiveness of a minimal psychological intervention. COPD: Journal of Chronic Obstructive Pulmonary Disease 2010;7(5):315-22.

\section{Livermore 2010a \{published data only\}}

Livermore N, Sharpe L, McKenzie D. Prevention of panic attacks and panic disorder in COPD. European Respiratory Journal 2010;35(3):554-63.

\section{Lolak 2008 \{published data only\}}

Lolak S, Connors G, Sheridan M, Shumway J, Hess S, Farrell S Effects of progressive relaxation on depression and anxiety in COPD patients. 158th Annual Meeting of the American Psychiatric Association. 2005

* Lolak S, Connors GL, Sheridan MJ, Wise TN. Effects of progressive muscle relaxation training on anxiety and depression in patients enrolled in an outpatient pulmonary rehabilitation program. Psychotherapy and Psychosomatics 2008;77(2):119-25.

\section{Pommer 2012 \{published data only\}}

* Pommer AM, Pouwer F, Denollet J, Pop VJM. Managing comorbid depression and anxiety in primary care patients with asthma and/or chronic obstructive pulmonary disease: study protocol for a randomised controlled trial. Trials 2012;13:6.

Pouwer F, Stoop CH. Disease management of depression and anxiety (DiMaCoDeA) in primary care patients with diabetes mellitus, asthma and/or COPD. www.trialregister.nl/trialreg/ admin/rctview.asp?TC=2626 2010 .

Williams 2011 \{published data only\}

Williams M. Can changing the way people with chronic lung disease think about breathlessness improve and sustain health outcomes?. www.anzctr.org.au/Trial/Registration/ TrialReview.aspx?id=336473 2011.

\section{Yang 2015 \{published data only\}}

Yang T. Evaluation, Psychological Intervention and Follow-up Study of Anxiety and Depression in Stable Chronic Obstructive 
Pulmonary Disease Patients. clinicaltrials.gov/ct2/show/ NCT02413840 2015.

\section{References to studies awaiting assessment}

Bove 2015 \{published data only\}

Bove DG. Dialogue aimed at reducing anxiety in patients with severe COPD (DIACOL). clinicaltrials.gov/ct2/show/ NCT02366390 2015.

* Bove DG, Overgaard D, Lomborg K, Lindhardt BO, Midtgaard J. Efficacy of a minimal home-based psychoeducative intervention versus usual care for managing anxiety and dyspnoea in patients with severe chronic obstructive pulmonary disease: a randomised controlled trial protocol. BMJ Open 2015;5:e008031.

\section{Cully 2012 \{published data only\}}

Cully JA, Armento ME, Mott J, Nadorff MR, Naik AD, Stanley MA. Brief cognitive behavioral therapy in primary care: a hybrid type 2 patient-randomised effectiveness-implementation design. Implementation Science 2012;7:64.

\section{Farver-Vestergaard 2014 \{published data only\}}

Farver-Vestergaard I. Mindfulness-based Cognitive Therapy for Chronic Obstructive Pulmonary Disease. clinicaltrials.gov/ct2/ show/NCT02042976 2014.

\section{Heslop 2013 \{published data only\}}

Heslop K. A single centre randomised controlled trial to identify if cognitive behavioural therapy delivered by respiratory nurses reduces anxiety and depression in patients with chronic obstructive pulmonary disease (CBT CARE Study). www.isrctn.com/ISRCTN55206395 2011.

* Heslop K, Newton J, Baker C, Burns G, Carrick-Sen D, De Soyza A. Effectiveness of cognitive behavioural therapy (CBT) interventions for anxiety in patients with chronic obstructive pulmonary disease (COPD) undertaken by respiratory nurses: the COPD CBT CARE study: (ISRCTN55206395). BMC Pulmonary Medicine 2013;13:62-8.

\section{Livermore 2015 \{published data only (unpublished sought but not} used)\}

Livermore N, Dimitri A, Sharpe L, McKenzie DK, Gandevia SC, Butler JE. Cognitive behaviour therapy reduces dyspnoea ratings in patients with chronic obstructive pulmonary disease (COPD). Respiratory Physiology \& Neurobiology 2015;216:35-42.

\section{Phan 2015 \{published and unpublished data\}}

Phan T, Carter O, Waterer G, Braithwaite M, Chung L, Rudd C, et al. Investigating the efficacy of cognitive behavioural therapy on improving the mental health of chronic obstructive pulmonary disease patients: a randomised control trial. Respirology. 2015; Vol. 20:35 (TO 057).

* Phan T, Carter O, Waterer G, Chung LP, Hawkins M, Rudd C. 'Breathing New Life: Investigating ways to improve the mental health of people living with chronic obstructive pulmonary disease' research study. Unpublished manuscript.
Shao 2003 \{published data only\}

Shao LZ. Effects of the behavioral intervention on the life quality of the patients with chronic obstructive pulmonary disease in remission period. Zhonghua Linchuang Kangfu Zazhi 2003;7(30):4078-9.

\section{References to ongoing studies}

ACTRN12612000254897 \{published data only\}

ACTRN12612000254897. Telephone Cognitive Behavioural Therapy for the treatment of depression and anxiety associated with chronic obstructive pulmonary disease: a pragmatic randomised controlled trial. www.anzctr.org.au/ ACTRN12612000254897.aspx 2012 (first received 14th of August 2015).

\section{ACTRN12614000915651 \{published data only\}}

ACTRN12614000915651. Randomised controlled trial of a brief telephone based cognitive behavioural therapy (CBT) for patients with chronic lung disease and anxiety and/ or depression undergoing pulmonary rehabilitation to evaluate the effect on quality of life, symptoms of anxiety and depression, and exacerbation rate. www.anzctr.org.au/ ACTRN12614000915651.aspx 2014 (first received 14th of August 2015).

\section{Additional references}

\section{Altman 1997}

Altman DG, Bland JM. Statistics notes. Units of analysis. BMJ 1997;314:1874.

\section{APA 1994}

American Psychiatric Association (APA). Diagnostic and Statistical Manual of Mental Disorders. 4th Edition. Washington, DC: American Psychiatric Association, 1994.

\section{Atlantis 2013}

Atlantis E, Fahey P, Cochrane B, Smith S. Bidirectional associations between clinically relevant depression or anxiety and COPD: a systematic review and meta-analysis. Chest 2013;144(3):766-77.

\section{BAI 1993}

Beck AT, Steer RA. Beck Anxiety Inventory: A Manual. 2nd Edition. San Antonio, TX: Psychological Corporation, 1993.

\section{Baraniak 2011}

Baraniak A, Sheffield D. The efficacy of psychologically based interventions to improve anxiety, depression and quality of life in COPD: a systematic review and meta-analysis. Patient Education and Counseling 2011;83:29-36. [DOI: 10.1016/ j.pec.2010.04.010]

\section{Beck 1961}

Beck AT, Ward CH, Mendelson M, Mock JE, Erbaugh JK. An inventory for measuring depression. Archives of General Psychiatry 1961;4:561-71. 


\section{Beck 1988}

Beck JG, Scott SK, Teague RB, Perez LI, Brown GA. Correlates of daily impairment in COPD. Rehabilitation Psychology 1988;33:77-84.

\section{Borg 1982}

Borg GA. Psychophysical basis of perceived exertion. Medicine and Science in Sports and Exercise 1982;14:377-81.

\section{Borson 1998}

Borson S, Claypoole K, McDonald GJ. Depression and chronic obstructive pulmonary disease: treatment trials. Seminars in Clinical Neuropsychiatry 1998;3:115-30.

\section{Breland 2015}

Breland JY, Hundt NE, Barrera TL, Mignogna J, Petersen NJ, Stanley MA. Identification of anxiety symptom clusters in patients with COPD: implications for assessment and treatment. International Journal of Behavioral Medicine 2015 [E-pub ahead of print]:1-8.

\section{Brenes 2003}

Brenes GA. Anxiety and chronic obstructive pulmonary disease: prevalence, impact and treatment. Psychosomatic Medicine 2003;65:963-70.

\section{Butland 1982}

Butland RJ, Pang J, Gross ER, Woodcock AA, Geddes DM. Two-, six-, and 12-minute walking tests in respiratory disease. British Medical Journal 1982;284(6329):1607-8.

\section{Campbell 2000}

Campbell M, Grimshaw J, Steen N. Sample size calculations for cluster-randomised trials. Changing Professional Practice in Europe Group (EU BIOMED II Concerted Action) Health Services Research Unit, University of Aberdeen, UK. Journal of Health Services Research and Policy Jan 2000;5(1):12-16.

\section{Clark 1986}

Clark D. A cognitive approach to panic. Behavior Research and Therapy 1986;24:461-70.

\section{Coventry 2007}

Coventry P, Hind D. Comprehensive pulmonary rehabilitation for anxiety and depression in adults with chronic obstructive pulmonary disease: a systematic review and meta-analysis. Journal of Psychosomatic Research 2007;63:551-65.

\section{Coventry 2013}

Coventry PA, Bower P, Keyworth C, Kenning C, Knopp J, Garrett C. The effect of complex interventions on depression and anxiety in chronic obstructive pulmonary disease: systematic review and meta-analysis. Plos One 2013;8(4):e60532.

\section{Davison 2003}

Davison GC, Neale JM. Abnormal Psychology. New York: John Wiley \& Sons, 2003.

\section{Deeks 2011}

Deeks JJ, Higgins JPT, Altman DG (editors). Chapter 9: Analysing data and undertaking meta-analyses. In: Higgins JPT, Green $\mathrm{S}$ (editors). Cochrane Handbook for Systematic Reviews of Interventions Version 5.1.0 (updated March 2011). The Cochrane Collaboration, 2011. Available from handbook.cochrane.org.

\section{Di Marco 2006}

Di Marco F, Verga M, Reggenta M, Maria Casanova F, Santus P, Blasi F, et al. Anxiety and depression in COPD patients: the roles of gender and disease severity. Respiratory Medicine 2006;100(10):1767-74.

\section{DiNicola 2013}

DiNicola G, Julian L, Gregorich SE, Blanc PD, Katz PP. The role of social support in anxiety for persons with COPD. Journal of Psychosomatic Research 2013;74(2):110-5.

\section{Eisner 2010}

Eisner MD, Blanc PD, Yelin EH, Katz PP, Sanchez G, Iribarren C, et al. Influence of anxiety on health outcomes in COPD. Thorax 2010;65:229-34.

\section{Elassal 2014}

Elassal G, Elsheikh M, Abu Zeid AG. Assessment of depression and anxiety symptoms in chronic obstructive pulmonary disease patients: a case-control study. Egyptian Journal of Chest Diseases and Tuberculosis 2014;63(3):575-82.

\section{Gillis 1995}

Gillis MM, Haaga DAF, Ford GT. Normative values for the Beck Anxiety Inventory, Fear Questionnaire, Penn State Worry Questionnaire, and Social Phobia and Anxiety Inventory. Psychological Assessment 1995;7(4):450-5.

\section{GOLD 2013}

Global Initiative for Chronic Obstructive Lung Disease Inc. Global strategy for the diagnosis, management and prevention of chronic obstructive pulmonary disease. www.goldcopd.org/ uploads/users/files/GOLD_AtAGlance_2013_Feb20.pdf (accessed 23 July 2013).

\section{GRADE 2013}

Schünemann H, Brożek J, Guyatt G, Oxman A, editor(s), GRADE working group. Handbook for grading the quality of evidence and the strength of recommendations using the GRADE approach. gdt.guidelinedevelopment.org/app/handbook/ handbook.html.

\section{Gudmundsson 2005}

Gudmundsson G, Gislason T, Janson C, Lindberg E, Hallin R, Ulrik CS, et al. Risk factors for rehospitalisation in COPD: role of health status, anxiety and depression. European Respiratory Journal 2005;26(3):414-9.

\section{Heslop-Marshall 2014}

Heslop-Marshall K, De Soyza A. Are we missing anxiety in people with chronic obstructive pulmonary disease (COPD)?. Annals of Depression and Anxiety 2014;1(5):1023. 


\section{Higgins 2003}

Higgins JPT, Thompson SG, Deeks JJ, Altman DG. Measuring inconsistency in meta-analyses. BMJ 2003;327:557-60.

\section{Higgins 2011a}

Higgins JPT, Altman DG, Sterne JAC (editors). Chapter 8: Assessing risk of bias in included studies. In: Higgins JPT, Green $\mathrm{S}$ (editors). Cochrane Handbook for Systematic Reviews of Interventions Version 5.1.0 (updated March 2011). The Cochrane Collaboration, 2011. Available from handbook.cochrane.org.

\section{Higgins 2011b}

Higgins JPT, Deeks JJ, Altman DG (editors). Chapter 16: Special topics in statistics. In: Higgins JPT, Green S (editors), Cochrane Handbook for Systematic Reviews of Interventions Version 5.1.0 (updated March 2011). The Cochrane Collaboration, 2011. Available from handbook.cochrane.org.

\section{Higgins 2011c}

Higgins JPT, Deeks JJ (editors). Chapter 7: Selecting studies and collecting data. In: Higgins JPT, Green S (editors), Cochrane Handbook for Systematic Reviews of Interventions Version 5.1.0 (updated March 2011). The Cochrane Collaboration, 2011. Available from handbook.cochrane.org.

\section{Jones 1991}

Jones PW, Quirk FH, Baveystock CM. The St George's Respiratory Questionnaire. Respiratory Medicine 1991;85 (Suppl B):25-31.

\section{Kandel 1998}

Kandel ER. A new intellectual framework for psychiatry. American Journal of Psychiatry 1998;155:457-69.

\section{Kaplan 2009}

Sadock BJ, Sadock VA, Ruiz P. Kaplan and Sadock's Comprehensive Textbook of Psychiatry. 9th Edition. Lippincott Williams \& Wilkins (LWW), 2009.

\section{Keil 2014}

Keil DC, Stenzel NM, Kuhl K, Vaske I, Mewes R, Rief W, et al. The impact of chronic obstructive pulmonary disease-related fears on disease-specific disability. Chronic Respiratory Disease 2014;11(1):31-40.

\section{Kerstjens 2001}

Kerstjens H, Groen H, Van Der Bij W. Respiratory medicine (clinical review: recent advances). BMJ 2001;323:1349-53.

\section{Kim 2000}

Kim HF, Kuic ME, Molinari VA, Hillman SL, Lalani S, Orengo CA, et al. Functional impairment in COPD patients: the impact of anxiety and depression. Psychosomatics 2000;41(6):465-71.

\section{Kunik 2001}

Kunik ME, Braun U, Stanley MA, Wristers K, Molinari V, Stoebner $D$, et al. One session cognitive behavioural therapy for elderly patients with chronic obstructive pulmonary disease. Psychological Medicine 2001;31:717-23.

\section{Laurin 2011}

Laurin C, Moullec G, Bacon SL, Lavoie KL. The impact of psychological distress on exacerbation rates in COPD. Therapeutic Advances in Respiratory Disease 2011;5(1):3-18. [DOI: $10.1177 / 1753465810382818]$

\section{Ley 1985}

Ley R. Agoraphobia, the panic attack and the hyperventilation syndrome. Behaviour Research and Therapy 1985;23:79-81.

\section{Livermore 2010b}

Livermore N, Sharpe L, McKenzie D. Panic attacks and panic disorder in chronic obstructive pulmonary disease: a cognitive behavioral perspective. Respiratory Medicine 2010;104(9):1246-53.

\section{Maurer 2008}

Maurer J, Rebbapragada V, Borson S, Goldstein R, Kunik ME, Yohannes AM, et al. Anxiety and depression in COPD. Chest 2008;134:43S-53S.

\section{Mikkelsen 2004}

Mikkelsen RL, Middelboe T, Pisinger C, Stage KB. Anxiety and depression in patients with chronic obstructive pulmonary disease (COPD). A review. Nordic Journal of Psychiatry 2004;58(1):65-70. [DOI: 10.1080/08039480310000824]

\section{NICE 2010}

National Institute of Clinical Excellence: Chronic obstructive pulmonary disease: management of chronic obstructive pulmonary disease in adults in primary and secondary care. 2010, London: National Clinical GuidelineGoogle Scholar.

\section{Norweg 2005}

Norweg AM, Whiteson J, Malgady R, Mola A, Rey M. The effectiveness of different combinations of pulmonary rehabilitation program components: a randomised controlled trial. Chest 2005;128:663-72.

\section{Nutt 1999}

Nutt DJ, Glue P, Lawsom CW, Wilson S. Flumazenil provocation of panic attacks: evidence for altered benzodiazepine receptor sensitivity in panic disorder. Archives of General Psychiatry 1999;47:917-25.

\section{Panagioti 2014}

Panagioti M, Scott C, Blakemore A, Coventry PA. Overview of the prevalence, impact, and management of depression and anxiety in chronic obstructive pulmonary disease. International Journal of COPD 2014;9:1289-306.

\section{Perna 2004}

Perna G, Caldirola D, Bellodi L. Panic disorder: from respiration to the homeostatic brain. Acta Neuropsychiatrica 2004;16:57-67.

\section{Rabe 2007}

Rabe KF, Hurd S, Anzueto A, Barnes PJ, Buist SA, Calverley P, et al. Global strategy for the diagnosis, management, and prevention of chronic obstructive pulmonary disease: GOLD executive summary. American Journal of Respiratory and Critical Care Medicine 2007;176:532-55. 


\section{RevMan 2014 [Computer program]}

Nordic Cochrane Centre, The Cochrane Collaboration. Review Manager 5 (RevMan 5). Version 5.3. Copenhagen: Nordic Cochrane Centre, The Cochrane Collaboration, 2014.

\section{Ries 1995}

Ries AL, Kaplan RM, Limberg TM, Prewitt LM. Effects of pulmonary rehabilitation of physiologic and psychosocial outcomes in patients with chronic obstructive pulmonary disease. Annals of Internal Medicine 1995;122:823-32.

\section{Rose 2002}

Rose C, Wallace L, Dickson R, Ayres J, Lehman R, Searle Y, et al. The most effective psychologically based treatments to reduce anxiety and panic in patients with chronic obstructive pulmonary disease (COPD): a systematic review. Patient Education and Counseling 2002;47:311-8. [PUBMED: 12135822]

\section{Royal College of Physcians}

Royal College of Physcians, Royal College of Psychiatrists. Smoking and mental health. Royal College of Physicians. Royal College of Physcians, 2013.

\section{Schünemann 2011}

Schünemann HJ, Oxman AD, Higgins JPT, Vist GE, Glasziou P, Guyatt GH. Chapter 11: Presenting results and 'Summary of findings' tables. In: Higgins JPT, Green S (editors), Cochrane Handbook for Systematic Reviews of Interventions Version 5.1.0 (updated March 2011). The Cochrane Collaboration, 2011. Available from handbook.cochrane.org.

\section{Smoler 1999}

Smoller J, Simon N, Polack M, Kradin R, Stern T. Anxiety in patients with pulmonary disease: comorbidity and treatment. Seminars in Clinical Neuropsychiatry 1999;4:84-97.

\section{Smoller 1996}

Smoller J, Pollack M, Otto M, Rosenbaum J, Kradin R. Panic, anxiety, dyspnea and respiratory disease. American Journal of Respiratory and Critical Care Medicine 1996;154:6-7.

\section{Spielberger 1970}

Spielberger CD, Gorsuch RL, Lushene RE. Manual for the StateTrait Anxiety Inventory. Palo Alto, CA: Consulting Psychologists Press, 1970.

\section{Sterne 2011}

Sterne JAC, Egger M, Moher D (editors). Chapter 10: Addressing reporting biases. In: Higgins JPT, Green S (editors). Cochrane Handbook for Systematic Reviews of Interventions. Version 5.1.0 (updated March 2011). The Cochrane Collaboration, 2011. Available from handbook.cochrane.org.

\section{Suh 2013}

Suh S, Ellis RJ, Sollers JJ 3rd, Thayer JF, Yang H, Emery CF. The effect of anxiety on heart rate variability, depression, and sleep in Chronic Obstructive Pulmonary Disease. Journal of Psychosomatic Research 2013;74:407-13.

\section{Tan 2013}

Tan SK, Benedicto J, Santiaguel J. Determinants of anxiety and depression among Filipino patients with chronic obstructive pulmonary disease: a multi-center study. European Respiratory Journal 2013;42(S57):4183.

\section{Usmani 2011}

Usmani ZA, Carson KV, Cheng JN, Esterman AJ, Smith BJ. Pharmacological interventions for the treatment of anxiety disorders in chronic obstructive pulmonary disease. Cochrane Database of Systematic Reviews 2011, Issue 11. [DOI: 10.1002/14651858.CD008483.pub2]

\section{Viinamaki 1998}

Viinamaki H, Kuikka J, Tiihonen J, Lehtonen J. Change in monoamine transporter density related to clinical recovery: a case control study. Nordic Journal of Psychiatry 1998;52:39-44.

\section{Weaver 1997}

Weaver TE, Richmond TS, Narsavage GL. An explanatory mode of functional status in chronic obstructive pulmonary disease. Nursing Research 1997;46:26-31.

\section{Willgoss 2011}

Willgoss T, Yohannes A, Goldbart J, Fatoye F. COPD and anxiety: its impact on patients' lives. Nursing Times 2011;107(15-16):16-9.

\section{Wu 2004}

Wu HS, Wu SC, Lin JG, Lin LC. Effectiveness of acupressure in improving dyspnoea in chronic obstructive pulmonary disease. Journal of Advanced Nursing 2004;45(3):252-9.

\section{Yohannes 2000}

Yohannes AM, Baldwin RC, Connolly MJ. Depression and anxiety in elderly outpatients with chronic obstructive pulmonary disease: prevalence, and validation of the BASDEC screening questionnaire. International Journal of Geriatric Psychiatry 2000;15(12):1090-6.

\section{Yohannes 2001}

Yohannes AM, Connolly MJ, Baldwin RC. A feasibility study of antidepressant drug therapy in depressed elderly patients with chronic obstructive pulmonary disease. International Journal of Geriatric Psychiatry 2001;16(5):451-4.

\section{Yohannnes 2006}

Yohannes A, Baldwin R, Connolly M. Depression and anxiety in elderly patients with chronic obstructive pulmonary disease. Age and Ageing 2006;35:457-9.

\section{Zigmond 1983}

Zigmond AS, Snaith RP. The Hospital Anxiety And Depression Scale. Acta Psychiatrica Scandinavia 1983;67:361-70.

\section{References to other published versions of this review Usmani 2013}

Usmani ZA, Carson KV, Heslop K, Esterman AJ, De Soyza A, Smith BJ. Psychological therapies for the treatment of 
anxiety disorders in chronic obstructive pulmonary disease. Cochrane Database of Systematic Reviews 2013, Issue 7. [DOI: 10.1002/14651858.CD010673]

* Indicates the major publication for the study

\section{CHARACTERISTICS OF STUDIES}

Characteristics of included studies [ordered by study ID]

de Godoy 2003

Methods
Design: a blinded prospective RCT
Multicentre?: no
Funders of the trial: supported by the Universidade de Caxias do Sul (BPC level II grant)
Duration of trial: October 1999-May 2001
Duration of participation: 12-week programme

Participants

Population description: people with COPD treated at a pulmonary rehabilitation clinic in Brazil; participant's COPD was stratified according to the Brazilian Society of Pulmonology and Tisiology guidelines into 3 severity levels: mild, moderate and severe

Setting: all participants were referred from the University's Department of Respiratory Diseases to the Pulmonary Rehabilitation Clinic

Inclusion criteria: diagnosis of COPD (corroborated by clinical history, physical examination, spirometry, chest plain films, thoracic computer tomography or both and pulse oximetry

Exclusion criteria: physical incapacity to perform the proposed protocol; refusal to participate in the pulmonary rehabilitation programme; lack of adherence to the pulmonary rehabilitation programme due to illness of more than 2 weeks' duration

Method of participant recruitment: pulmonary rehabilitation clinic patients

Total number randomised: 30 participants

Withdrawals and exclusions: no withdrawals mentioned

Age: mean age 60.33

Sex: 22 men and 8 women

Race/ethnicity: not stated

Interventions

\section{Intervention}

Number randomised to group: 14

Details of the interventions:

- 12 psychotherapy sessions including sessions of psychotherapy which addressed the patient's psychosocial needs and included his/her social, marital, work, health, and interpersonal philosophy and habits; cognitive therapy and logotherapy techniques were used during these sessions

Intervention intensity: 12-week treatment programme with 12 psychotherapy sessions in addition to co-interventions of 24 physiotherapy sessions, 24 physical exercise sessions and 3 educational sessions

Mode of delivery: unclear if intervention was delivered in group settings or to individuals

\section{Co-interventions:}


- 24 sessions of exercise (arm and leg exercises, aerobic condition with a treadmill, and flexibility training

- 24 sessions of physiotherapy (diaphragmatic breathing, sputum clearance, bending forward postures, pursed-lip breathing, postural drainage, chest percussion, vibration, directed cough and forced expiratory technique was used)

- 3 education sessions (including compliance, coping with illness, stress management, anatomy and physiology of the lungs, relaxation and energy-saving techniques, drug actions, side effects and technique for administration, good nutrition, use of oxygen, environmental control, intimacy and sexuality, the doctor-patient relationship, smoking cessation, benefits of exercise

\section{Comparison}

Comparison name: reported as 'Group 2' in the paper

Number randomised to group: 16

Details of the interventions:

- 24 sessions of exercise (arm and leg exercises, aerobic condition with a treadmill, and flexibility training)

- 24 sessions of physiotherapy (diaphragmatic breathing, sputum clearance, bending forward postures, pursed-lip breathing, postural drainage, chest percussion, vibration, directed cough and forced expiratory technique was used)

- 3 education sessions (including compliance, coping with illness, stress management, anatomy and physiology of the lungs, relaxation and energy-saving techniques, drug actions, side effects and technique for administration, good nutrition, use of oxygen, environmental control, intimacy and sexuality, the doctor-patient relationship, smoking cessation, benefits of exercise

Intervention intensity: 12-week treatment programme with 24 physiotherapy sessions, 24 physical exercise sessions and 3 educational sessions

Mode of delivery: unclear if intervention was delivered in group settings or to individuals

Outcomes collected: BAI, BDI and 6MWD
Time points measured: baseline and post-intervention
Person collecting time point: not specified
Outcome measures validated?: yes for all three
Missing data addressed?: not reported
Note: no mention of a sample size calculation is made but authors do report the "relatively small sam-
ple size" as a limitation of the study.
$\begin{aligned} & \text { Funding: Supported by the Universidade de Caxias do Sul (BPC level II grant); No commercial party hav- } \\ & \text { ing a direct financial interest in the results of the research supporting this article has or will confer a } \\ & \text { benefit upon the authors or upon any organization with which the authors are associated }\end{aligned}$

\section{Risk of bias}

\begin{tabular}{lll}
\hline Bias & Authors' judgement & Support for judgement \\
\hline $\begin{array}{l}\text { Random sequence genera- } \\
\text { tion (selection bias) }\end{array}$ & Unclear risk & $\begin{array}{l}\text { Method of randomisation not specified "patients were randomised into 2 } \\
\text { groups" }\end{array}$ \\
\hline $\begin{array}{l}\text { Allocation concealment } \\
\text { (selection bias) }\end{array}$ & Unclear risk & Details of allocation concealment not reported \\
\hline
\end{tabular}


de Godoy 2003 (Continued)

Blinding of participants and personnel (performance bias)

All outcomes

\begin{tabular}{lll}
\hline Blinding of outcome as- & Unclear risk & No mention of blinding for outcome assessors \\
sessment (detection bias) &
\end{tabular}

All outcomes

Incomplete outcome data Low risk (attrition bias)

All outcomes

Selective reporting (re- Unclearisk

Selective reporting (re- Unclear risk porting bias)
Reasons for choosing not to participate prior to randomisation ( $\mathrm{n}=6$ participants) and attrition post recruitment ( $n=3$ intervention participants) were reported for all participants

Other bias Low risk No other biases identified

Hynninen 2010

Country: Norway
Design: prospective RCT
Multicentre?: no
Funders of the trial: no funding sources mentioned
Duration of trial: participants were enrolled over a period of 1.5 years; no other information provided
Duration of participation: 8 months from baseline

Participants

Population description: people with COPD who answered positively to at least 2 of the 5 anxiety and depression questions from the PRIME-MD questionnaire

Setting: participants in both groups attended the pulmonary clinic at baseline and 2 and 8 months later for spirometry, self-report measures and provision of Actigraph device for sleep registration

Inclusion criteria: COPD diagnosis confirmed with post -bronchodilator spirometry FEV $1<80 \%$ predicted and ratio $<0.7$, aged 40 years or over, had BAI scores $>15$ and/or BDI-II > 13, not participating in other psychological interventions e.g. pulmonary rehabilitation, no cognitive impairment (MMSE score > 23) and no severe psychiatric disorders as per DSMIV

Exclusion criteria: as per inclusion criteria

Method of participant recruitment: consecutive eligible patients who were interested in participating in the study were recruited from an outpatient clinic at the Haukeland University Hospital, Bergen, Norway or were recruited via a newspaper advertisement

Total number randomised: 51 participants

Withdrawals and exclusions: intervention arm: 2 discontinued intervention; control arm: 2 could not be contacted and 1 died

Age: intervention group: mean 59.3 years; control group: mean 62.6 years

Sex: intervention group: 11 women, 14 men; control group: 15 women, 11 men

Race/ethnicity: not reported 
Hynninen 2010 (Continued)

Interventions

\section{Intervention}

Number randomised to group: 25 (23 received allocated intervention)

Details of the interventions:

- psychoeducation awareness (how COPD may affect psychological well-being, patterns of anxiety and depression adding to the burden of lung disease)

- relaxation (using breathing techniques and postural changes for relaxation and coping with physical symptoms)

- cognitive therapy (identifying unhelpful thoughts and explore more functional patterns of thought)

- behavioural therapy (identify depressive, passive behaviours and replace them with activities that are pleasant/increase mastery;

- fear-based exposure (replace avoidance with graded exposure to increase tolerance)

- sleep skills management (learn about sleep hygiene and use skills);

CBT was undertaken in the Department of Clinical psychology. The group session was facilitated by two masters-level psychology students; The sessions were videotaped and a specialist in clinical psychology monitored the students' competence

Intervention intensity: 7 weekly 2 -h sessions

Mode of delivery: group sessions (4-6 participants, 5 on average)

\section{Co-interventions}

- Participants were phoned 1 and 3 months after post-treatment assessments and encouraged to maintain behavioural changes instigated in therapy

\section{Comparison}

Comparison name: Enhanced Standard care for COPD

Number randomised to group: 26 (23 received allocated intervention)

Details of the interventions:

- standard COPD care plus telephone contact every 2 weeks in the intervention period of seven weeks;

- the same student therapists conducted the phone calls

Intervention intensity: phone calls every 2 weeks for intervention period; Calls lasted 5-10 min

Mode of delivery: telephone

\section{Co-interventions}

- participants were phoned 1 and 3 months after post-treatment assessments and encouraged to maintain behavioural changes instigated in therapy

Outcomes

Outcomes collected: BAI, BDI, SGRQ, PSQI, Actigraphy (objective measure of sleep efficiency) and CSQ

Time points measured: baseline, 2 and 8 months (or baseline, post treatment and six months post treatment)

Person collecting time point: not reported

Outcome measures validated?: yes for all

Missing data addressed?: yes, ITT analysis used and authors report that missing data at one measurement point did not prevent including the individual in the analysis

Notes

Note: the target sample size identified as being necessary in the power analysis ( 33 in each arm) was not reached by the end of the study period ( 25 in the intervention arm and 26 in the control arm) 
Hynninen 2010 (Continued)

Funding: no mention of funding or financial support for this work

\section{Risk of bias}

\begin{tabular}{|c|c|c|}
\hline Bias & Authors' judgement & Support for judgement \\
\hline $\begin{array}{l}\text { Random sequence genera- } \\
\text { tion (selection bias) }\end{array}$ & Unclear risk & Randomisation reported however methods not described \\
\hline $\begin{array}{l}\text { Allocation concealment } \\
\text { (selection bias) }\end{array}$ & Low risk & $\begin{array}{l}\text { Allocation concealment was implemented using numbered containers that } \\
\text { were identical in appearance for the two groups }\end{array}$ \\
\hline $\begin{array}{l}\text { Blinding of participants } \\
\text { and personnel (perfor- } \\
\text { mance bias) } \\
\text { All outcomes }\end{array}$ & High risk & Neither participants nor therapists were blinded to the intervention \\
\hline $\begin{array}{l}\text { Blinding of outcome as- } \\
\text { sessment (detection bias) } \\
\text { All outcomes }\end{array}$ & Unclear risk & No mention of blinding for outcome assessors \\
\hline $\begin{array}{l}\text { Incomplete outcome data } \\
\text { (attrition bias) } \\
\text { All outcomes }\end{array}$ & Low risk & $\begin{array}{l}\text { Reasons for attrition reported in participant flow chart. Missing outcome da- } \\
\text { ta at one measurement point did not preclude analysis as mixed models with } \\
\text { random effect was used for analysis; Intention-to-treat analysis occurred }\end{array}$ \\
\hline $\begin{array}{l}\text { Selective reporting (re- } \\
\text { porting bias) }\end{array}$ & Low risk & Prespecified protocol available and no selective reporting identified \\
\hline Other bias & Low risk & No other biases identified \\
\hline
\end{tabular}

Kunik 2008

$\begin{array}{ll}\text { Methods } & \text { Country: USA } \\ \text { Design: prospective RCT } \\ \text { Multicentre?: no } \\ \text { Funders of the trial: grant from Department of Veterans Affairs, Veterans Health Administration, Health } \\ \text { Services Research and Development; Houston Centre for Quality Care and Utilization Studies and } \\ \text { South Central Medical Illness Research, Education and Clinic Center, Department of Veterans Affairs } \\ \text { Duration of trial: } 11 \text { July 2002-30 April } 2005 \\ \text { Duration of participation: } 12 \text { months' follow-up from baseline } \\ \text { Population description: people with a chronic breathing disorder (COPD) who had moderate anxiety } \\ \text { symptoms and/or depression and were receiving care at the Michael E DeBakey VA Medical Centre } \\ \text { (MEDVAMC) within the year before the study } \\ \text { Setting: participants were from the Michael E DeBakey VA Medical Center in Houston, Texas, USA and } \\ \text { community members meeting the criteria outside of the medical centre } \\ \text { Inclusion criteria: diagnosis of COPD confirmed with spirometry (ratio < 70 and FEV1 < 70; according to } \\ \text { ATS 1991); moderate anxiety (BAI } \geq 16 \text { ) and/or depression (BDI > 14); and treatment by primary care or } \\ \text { provider or pulmonologist } \\ \text { Exclusion criteria: cognitive disorder or evidence of score of 23 or less on MMSE, a psychotic disorder } \\ \text { and people with psychotic and non-nicotine substance use disorders }\end{array}$

Psychological therapies for the treatment of anxiety disorders in chronic obstructive pulmonary disease (Review) 
Method of participant recruitment: people on administrative database from the Michael DeBakey Medical Center (MEDVAMC) were targeted for recruitment and screened, in addition to other methods including flyers and advertisements

Total number randomised: 238

Withdrawals and exclusions: 69 participants dropped out following randomisation(because of the following reasons: medical 2, no time 6,transportation 6,no interest 49, no information 46).

Age: Mean $66.3 \pm 10.2$ years

Sex: 226 men and 9 women in total

Race/ethnicity: white $\mathrm{n}=192$, Hispanic $\mathrm{n}=7$ and black $\mathrm{n}=38$

Interventions

\section{Intervention}

Number randomised to group: 118 (89 attended at least one CBT session)

Details of the interventions:

- education and awareness training focused on anxiety, depression and associated physiological, cognitive and behavioural symptoms (session 1)

- relaxation training (session 2)

- increasing pleasurable activity and decreasing anxiety-related avoidance (session 2-3)

- cognitive therapy - alternative thoughts, encouraging self statements and thought stopping (session 4-5)

- problem-solving techniques (session 6)

- sleep management skills (session 7)

- skills review and maintenance of gains (session 8)

Intervention intensity: eight 1-h sessions

Mode of delivery: group CBT (up to 10 participants each session)

\section{Co-interventions}

- Group discussion

\section{Comparison}

Comparison name: COPD Education

Number randomised to group: 120 (92 received the education intervention)

Details of the interventions:

- COPD education 45 min sessions with similar frequency to CBT group;

- Topics included: breathing strategies, airways management, physiology of lung disease, use of oxygen, avoidance of environmental irritants, nutrition, exercise, smoking cessation and end-of-life planning

Intervention intensity: eight 45-min lectures and 15-min discussions (to control for contact time)

Mode of delivery: group sessions

\section{Co-interventions:}

- Group discussion

Outcomes

Outcomes collected: CRQ, SF36, BAI, BDI, 6MWD and service use determined by number of hospitalisations, outpatient, mental health and emergency room visits

Time points measured: 1 month, 2 months, 4 months, 8 months and 12 months 
Kunik 2008 (Continued)

Person collecting time point: not reported

Outcome measures validated?: yes, with the exception of service use outcomes

Missing data addressed?: Yes, ITT analysis used and authors reported that missing data at one measurement point did not prevent including the individual in the analysis

Notes

Note: of 256 eligible participants, 238 were randomised but only 181 attended their first session. Study authors reported that retention in research studies at that particular facility could often be challenging with patients treated at Veteran Affairs facilities having more physical and mental health problems than the average US citizen. Also, the sample size calculation in the statistical analysis section stated that 120 participants per group would be required yet this $n$-value was not met

Funding: study supported by grant No IIR 00-097 from the Department of Veterans Affairs, Veteran Health Administration, Health Services Research and Development, Washington DC and in part by Houston Center for Quality of Care and Utilization Studies, Office of Research and Development; and the South Central Mental IIlness Research, Education and Clinical Center, Department of Veteran Affairs

\section{Risk of bias}

\begin{tabular}{lll}
\hline Bias & Authors' judgement & Support for judgement \\
\hline $\begin{array}{l}\text { Random sequence genera- } \\
\text { tion (selection bias) }\end{array}$ & Low risk & $\begin{array}{l}\text { Randomisation list occurred via computer program (SAS) with blocks to pro- } \\
\text { vide appropriately equal numbers per class of COPD; instructor assigned treat- } \\
\text { ment to the code initially by flip of coin }\end{array}$ \\
\hline $\begin{array}{l}\text { Allocation concealment } \\
\text { (selection bias) }\end{array}$ & Low risk & $\begin{array}{l}\text { The statistician provided randomisation numbers and treatment codes to the } \\
\text { study co-ordinator when sufficient participants for two classes had completed } \\
\text { the baseline assessment and consented to participate; the instructor assigned } \\
\text { the treatment to the code initially by flipping a coin }\end{array}$ \\
\hline
\end{tabular}

\begin{tabular}{|c|c|c|}
\hline $\begin{array}{l}\text { Blinding of participants } \\
\text { and personnel (perfor- }\end{array}$ & High risk & $\begin{array}{l}\text { Participants and staff performing the intervention were not blinded to treat- } \\
\text { ment allocation }\end{array}$ \\
\hline
\end{tabular}

mance bias)

All outcomes

\begin{tabular}{lll}
$\begin{array}{l}\text { Blinding of outcome as- } \\
\text { sessment (detection bias) } \\
\text { All outcomes }\end{array}$ & Low risk & Study personnel performing assessments were blinded to treatment condition \\
\hline $\begin{array}{l}\text { Incomplete outcome data } \\
\begin{array}{l}\text { (attrition bias) } \\
\text { All outcomes }\end{array}\end{array}$ & Low risk & $\begin{array}{l}\text { Missing data accounted for in regression analyses; reasons for participants' ex- } \\
\text { clusion (pre-enrolment) and attrition of participants post recruitment are re- } \\
\text { ported in detail within the subject flowchart; reasons for exclusion reported }\end{array}$
\end{tabular}

\begin{tabular}{lll}
\hline $\begin{array}{l}\text { Selective reporting (re- } \\
\text { porting bias) }\end{array}$ & Low risk & Pre-specified protocol available and no selective reporting identified \\
\hline Other bias & Low risk & No other biases identified \\
\hline
\end{tabular}

6MWD: Six Minute Walking Distance

BAl: Beck Anxiety Inventory

BDI: Beck Depression Inventory

COPD: chronic obstructive pulmonary disease

CRQ: Chronic Respiratory Questionnaire

CSQ: client satisfaction questionnaire

DSMIV: Diagnostic and Statistical Manual of Mental Disorders (4th Ed)

FEV1: forced expiratory volume in 1 second

ITT: intention-to-treat

MMSE: Mini-Mental State Examination 
PRIME-MD: Primary Care Evaluation of Mental Disorders

PSQI: Pittsburgh Sleep Quality Index

$\mathrm{RCT}$ : randomised controlled trial

SGRQ: Saint George's Respiratory Questionnaire

SF36: Medical Outcomes Survey Short Form 36

Characteristics of excluded studies [ordered by study ID]

\begin{tabular}{|c|c|}
\hline Study & Reason for exclusion \\
\hline Aubuchon 1990 & Participants not diagnosed with anxiety at baseline \\
\hline Blake 1990 & Participants not diagnosed with anxiety at baseline (diagnosed with stress) and not COPD-specific \\
\hline Blumenthal 2014 & Participants not diagnosed with anxiety at baseline \\
\hline Cully 2007 & Not a RCT as defined for this review \\
\hline de Godoy 2005 & $\begin{array}{l}\text { No adequate control group: co-intervention in groups } 1 \text { and } 3 \text { was an exercise regimen, which was } \\
\text { not a prespecified co-intervention for this review }\end{array}$ \\
\hline Doyle 2013 & Participants not diagnosed with anxiety at baseline. No intervention arm \\
\hline Gift 1992 & Participants not diagnosed with anxiety at baseline \\
\hline Lamers 2010 & Anxiety data not reported separately \\
\hline Livermore 2010a & Participants not diagnosed with anxiety at baseline and mean HADS scores are with normal range \\
\hline Lolak 2008 & Progressive muscle relaxation, not psychological intervention \\
\hline Pommer 2012 & Multi-component intervention, more than just a psychological intervention \\
\hline Williams 2011 & Participants not diagnosed with anxiety at baseline \\
\hline Yang 2015 & Multi-component intervention, more than just a psychological intervention \\
\hline
\end{tabular}

COPD: chronic obstructive pulmonary disease

HADS: Hospital Anxiety and Depression Scale

RCT: randomised controlled trial

Characteristics of studies awaiting assessment [ordered by study ID]

Bove 2015

\begin{tabular}{ll}
\hline Methods & Parallel RCT \\
\hline Participants & $\mathrm{N}=66$ subjects with severe COPD and associated anxiety randomised \\
& $\begin{array}{l}\text { Exclusion criteria: HADS-A (anxiety) subscale score of }<8, \text { a psychiatric diagnosis, pulmonary can- } \\
\text { cer or involvement in a different interventional trial }\end{array}$ \\
\hline Interventions & $\begin{array}{l}\text { Single psycho-educative session in the participant's home in combination with a telephone boost- } \\
\text { er session; intervention based on a manual, with theoretical foundation in CBT and psychoeduca- } \\
\text { tion } \\
\text { Usual care comparator }\end{array}$
\end{tabular}

Psychological therapies for the treatment of anxiety disorders in chronic obstructive pulmonary disease (Review) 
Bove 2015 (Continued)

Outcomes Primary outcomes: HADS-A (anxiety) and HADS-D (depression)

Secondary outcomes: CRQ and SGRQ for quality of life and mastery of dyspnoea

Notes Part of a PhD project

\section{Cully 2012}

\begin{tabular}{ll}
\hline Methods & Parallel RCT \\
\hline Participants & $\begin{array}{l}\mathrm{N}=320 \text { participants with patient-reported functional limitations associated with COPD, and/or } \\
\text { heart failure, with clinically significant symptoms of anxiety and/or depression }\end{array}$ \\
& $\begin{array}{l}\text { Exclusion criteria: clinical factors e.g. ongoing psychotherapy, concurrent speciality mental health- } \\
\text { care and patient factors e.g. cognitive, bipolar, psychotic or substance abuse disorders }\end{array}$
\end{tabular}

Interventions

Brief manualised CBT delivered by clinicians in comparison to usual care

Intervention group: 6 weekly treatment sessions and 2 brief (10-15-min) telephone booster sessions within a 4-month time frame of the ACCESS

Intervention: core modules focus on increasing awareness and controlling physical and emotional symptoms and subsequently producing skills aligning with their most pressing needs; therapists used a structured manual yet could also tailor the intervention with the participant based on their needs; participant workbook also provided

Control group: usual care with feedback about their depression and anxiety

\begin{tabular}{ll}
\hline Outcomes & Participant outcomes: depression, anxiety and physical health functioning \\
& $\begin{array}{l}\text { Implementation outcomes: participant engagement, adherence and clinician brief CBT adoption } \\
\text { and fidelity }\end{array}$
\end{tabular}

\section{Notes}

\section{Farver-Vestergaard 2014}

\begin{tabular}{ll}
\hline Methods & Double-blind RCT \\
\hline Participants & $\begin{array}{l}\text { Estimated } n=120 \text { participants with severe to very severe COPD, motivated to participate in pul- } \\
\text { monary rehabilitation and with sufficient mobility to attend pulmonary rehabilitation }\end{array}$ \\
& $\begin{array}{l}\text { Exclusion criteria: certain co-morbidities (e.g. unstable coronary complications, psychiatric illness), } \\
\text { severe cognitive disability (e.g. dementia) and inability to speak Danish }\end{array}$ \\
\hline Interventions & $\begin{array}{l}\text { Mindfulness-based cognitive therapy + pulmonary rehabilitation compared to pulmonary rehabili- } \\
\text { tation only }\end{array}$ \\
& $\begin{array}{l}\text { Intervention group: 8-week manual-based programme developed by Segal, Williams and Teasdale } \\
\text { (2013) adjusted to the COPD population; programme delivered as an add-on to an 8-week stan- } \\
\text { dardised rehabilitation programme consisting of physical exercise and COPD-specific patient edu- } \\
\text { cation } \\
\text { Control group: 8-week standardised rehabilitation programme consisting of physical exercise and } \\
\text { COPD-specific patient education }\end{array}$ \\
\end{tabular}


Farver-Vestergaard 2014 (Continued)

Outcomes

Primary outcomes: quality of life (CAT), anxiety and depression (HADS), and BODE index

Secondary outcomes: physical activity (measured by accelerometry), inflammation and oxidative stress (measured by gene expression profiling)

Notes

Heslop 2013

\begin{tabular}{|c|c|}
\hline Methods & Parallel RCT \\
\hline \multirow[t]{2}{*}{ Participants } & $\begin{array}{l}\mathrm{N}=312 \text { participants with a confirmed diagnosis of COPD (FVC/FEV1 ratio }<70 \% \text {, (NICE } 2010) \text {; in- } \\
\text { cluding mild-moderate (FEV } 1>50 \% \text { predicted) and severe-very severe }(<50 \% \text { predicted)), proba- } \\
\text { ble anxiety defined by HADSA }>8 \text {, willing to participate and provide informed consent, agreed to } \\
\text { attend minimum of } 2 \text { and maximum of } 6 \text { CBT sessions }\end{array}$ \\
\hline & $\begin{array}{l}\text { Exclusion criteria: people with HADS-A scores }<8 \text {, known psychosis or personality disorders, cur- } \\
\text { rently receiving psychological therapy including counselling or psychotherapy, unable to engage in } \\
\text { CBT (due to cognitive impairment or dementia) and limited verbal and/or written communication } \\
\text { problems }\end{array}$ \\
\hline
\end{tabular}

Interventions

Psychological treatment for anxiety and depression through CBT compared to self-help leaflets

Intervention group: 2-6 sessions depending on participant need and progress as per HADS (usually involved 1 session of CBT every two weeks); components of CBT included developing a CBT formulation, psychoeducation about COPD with panic/depression, identifying/challenging negative or unhelpful thinking, identifying challenging negative or unhelpful behaviours, distraction, breathing control, relaxation, mindfulness, behavioural experiments and graded exposure

Control group: self-help leaflets

Outcomes Primary outcome: HADS (3 months)

Secondary outcomes: HADS (6 and 12 months)

Notes

Livermore 2015

\begin{tabular}{ll}
\hline Methods & RCT \\
\hline Participants & People with moderate to severe COPD \\
\hline Interventions & CBT (4 sessions) versus usual care \\
\hline Outcomes & Dyspnea scores \\
\hline Notes & $\begin{array}{l}\text { We have tried to contact the study authors to get more information in regards to the baseline anxi- } \\
\text { ety scores of the individual participants to assess the eligibility of some of the participants for this } \\
\text { review. We will continue intermittent attempts at contact and if no response has been received by } \\
\text { the time of the next update, this study will be moved to the excluded category. }\end{array}$
\end{tabular}


Phan 2015

\begin{tabular}{ll}
\hline Methods & Parallel, multi-arm RCT \\
\hline Participants & $\begin{array}{l}\mathrm{N}=128 \text { people attending the COPD outpatient community clinics in Perth, Western Australia were } \\
\text { included if they had a diagnosis of COPD confirmed from medical records and screened positive for } \\
\text { anxiety and depression }\end{array}$
\end{tabular}
anxiety and depression

Exclusion criteria: life expectancy of less than 6 months, were currently involved in another research study, had an illness exacerbation resulting in hospitalisation within the previous month, were not fluent in English, or were blind, deaf or diagnosed with dementia or Alzheimer's disease

Interventions

Six weeks of 2 formats of CBT, being group therapy (6-16 people in each group) versus a self-paced simulation-based learning resource (DVD) compared to usual care; group therapy consisted of 2 half-day sessions a fortnight apart and a 1-hour telephone booster session 4 weeks later; the sessions were semi-structured in nature and included both CBT global concepts and those that were specific to people with COPD; a manual was provided to participants for referral to CBT concepts; CBT included information about treatment rationale explaining the link between cognitions, behaviours and breathing, coping skills training, cognitive restructuring and the application and maintenance of learned coping skills; The self-paced simulation included 6 vignettes (participants were asked to watch one per week) approximately 10 min in length on CBT skills to cope with anxiety disorders and depression, a resource manual to guide participants through the video and a weekly phone call by a researcher to check if each vignette was watched, activities completed and if participants had any questions

Usual care group was under the usual treating physician; telephone follow-up (or home visits for those with hearing difficulties) occurred at one week post-intervention completion, 3, 6, 9 and 12 months

Outcomes Primary outcomes: BAI and BDI

Secondary outcomes: SGRQ, absolute FEV1, FEV1 \% predicted, FVC

Notes

Shao 2003

\begin{tabular}{|c|c|}
\hline Methods & $\begin{array}{l}\text { The aim of the study was to explore the effect of behavioral intervention on the quality of life } \\
\text { among people with COPD during the remission period }\end{array}$ \\
\hline Participants & 54 people with COPD were randomly divided into intervention group and control group \\
\hline Interventions & $\begin{array}{l}\text { The } 2 \text { groups were treated with the same clinical therapy. The intervention group was also given a } \\
\text { behavioral intervention that included psychological therapy with a somatic function and lifestyle } \\
\text { intervention }\end{array}$ \\
\hline Outcomes & $\begin{array}{l}\text { All participants were evaluated with the Fang Zhong-Jun quality-of-life scale pretreatment, before } \\
\text { discharge, } 3 \text { months and } 1 \text { year after discharge }\end{array}$ \\
\hline Notes & $\begin{array}{l}\text { Results: at } 1 \text { year follow-up, quality of life (measured as 'ability of daily life' and 'status of social ac- } \\
\text { tivity'), psychological symptom of depression, and psychological symptom of anxiety in the inter- } \\
\text { vention group }(2.03+/-0.32,2.29+/-0.77,2.36+/-0.34,2.07+/-0.25) \text { were significantly lower than } \\
\text { those in the control group }(2.29+/-0.30,2.39+/-0.41,2.41+/-0.28,2.16+/-0.51),(t=2.801,2.914, P \\
<0.01, t=2.250,2.340, P<0.05)\end{array}$ \\
\hline
\end{tabular}

ACCESS: Adjusting to Chronic Conditions Using Eucation, Support, and Skills

BAl: Beck Anxiety Inventory

BDI: Beck Depression Inventory

Psychological therapies for the treatment of anxiety disorders in chronic obstructive pulmonary disease (Review) 
BODE: Body mass index, airflow Obstruction, Dyspnea and Exercise capacity

CAT: COPD Assessment Text

CBT: cognitive behavioural therapy

COPD: chronic obstructive pulmonary disease

CRQ: Chronic Respiratory Questionnaire

HADS-A: Hospital Anxiety and Depression Scale - Anxiety Subscale

HADS-D: Hospital Anxiety and Depression Scale - Depression Subscale

FEV1: forced expiratory volume in 1 second

FVC: forced vital capacity

$\mathrm{RCT}$ : randomised controlled trial

SGRQ: Saint George's Respiratory Questionnaire

Characteristics of ongoing studies [ordered by study ID]

\section{ACTRN12612000254897}

Trial name or title

Telephone Cognitive Behavioural Therapy for the treatment of depression and anxiety associated with chronic obstructive pulmonary disease: a pragmatic randomised controlled trial

\begin{tabular}{ll}
\hline Methods & Parallel RCT \\
\hline Participants & $\begin{array}{l}\mathrm{N}=140 \text { participants over } 45 \text { years of age, with a HADS score of }>8 \text { and a PHQ-9 score }>10, \text { with a di- } \\
\text { agnosis of COPD, living in the community and able to speak English }\end{array}$ \\
& $\begin{array}{l}\text { Exclusion criteria: people who commenced anti-depressants and/or anxiolytics in the past } 3 \\
\text { months or have had a clinically significant change in this medication in the last } 3 \text { months and deaf- } \\
\text { ness }\end{array}$
\end{tabular}

Interventions

Telephone administered Cognitive Behavioural Therapy (CBT) plus usual care

Control population not specified

Intervention group: starts with a face-to-face 50-min session at the outpatient clinic or participant's home followed by 8 scheduled weekly telephone calls for up to $30 \mathrm{~min}$ in length

Intervention includes behavioural strategies such as behavioural activation, activity scheduling, relaxation training, exposure hierarchies and social skills training, as well as cognitive strategies, such as cognitive restructuring, structured problem solving and behavioural experiments

Outcomes Primary outcomes: BAI and PHQ-9 (depression scale)

Secondary outcomes: costs of illness (including medical treatment, equipment and working hours), quality of life (AQoL-4D), client satisfaction, COPD assessment test, General Self-Efficacy scale, Working Alliance Inventory, acute hospitalisations, number of pulmonary rehabilitation attendances

Starting date February 2012

Contact information

Colleen Doyle; +61 38387 2169; c.doyle@nari.unimelb.edu.au; National Ageing Research Institute 34-54 Poplar Rd Parkville Victoria 3052, Australia

\section{Notes}

\section{ACTRN12614000915651}

Trial name or title

Randomised controlled trial of a brief telephone based cognitive behavioural therapy (CBT) for patients with chronic lung disease and anxiety and/or depression undergoing pulmonary rehabilitation to evaluate the effect on quality of life, symptoms of anxiety and depression, and exacerbation rate 
ACTRN12614000915651 (Continued)
Methods
Parallel RCT

Participants

$\mathrm{N}=100$ participants with chronic lung disease, undergoing pulmonary rehabilitation and clinical or sub-clinical anxiety or depression defined by GAI score $\geq 4 / 20$, and/or GDS of $\geq 4 / 15$

Exclusion criteria: inability to provide written informed consent, known psychotic disorder, cognitive impairment determined by MOCA score of $<25 / 30$ and current enrolment in other interventional clinical trials that would potentially interfere with this study

Interventions
Intervention group: 6 CBT sessions divided into: 2 face-to-face individual sessions of $1 \mathrm{~h}$ each, with-
in the first 4 weeks of pulmonary rehabilitation; 4 phone sessions of 60 min each undertaken for
counselling, each session will be fortnightly within the first 2 months after the face-to-face sessions;
CBT intervention will be standardised following a manual written by the Prince Charles Hospital
psychology department
Control group: usual care comprised of medical treatment and pulmonary rehabilitation

Outcomes Primary outcomes: symptoms of anxiety using GAI and depression using GDS

Secondary outcomes: 6MWD, SGRQ, Asthma Quality-of-Life Questionnaire, Asthma Control Questionnaire, primary care and hospital health service utilisation, pulmonary rehabilitation attendance and participation assessment

\begin{tabular}{ll}
\hline Starting date & September 2014 \\
\hline Contact information & $\begin{array}{l}\text { A/Prof lan Yang; +61-7-31395050; lan.Yang@health.qld.gov.au; The Prince Charles Hospital Rode } \\
\text { Road Chermside, Postcode 4032 Queensland, Australia; and Dr Marsus I Pumar; +61 04 } 37739874 ; \\
\text { marsusicaro@gmail.com; The Prince Charles Hospital Rode Road Chermside, Postcode } 4032 \\
\text { Queensland, Australia }\end{array}$ \\
\hline
\end{tabular}

Notes

6MWD: Six Minute Walking Distance

BAI: Beck Anxiety Inventory

CBT: cognitive behavioural therapy

COPD: chronic obstructive pulmonary disorder

GAI: Geriatric Anxiety Inventory

GDS: Geriatric Depression scale

HADS: Hospital Anxiety and Depression Scale

MOCA: Montreal Cognitive Assessment (MOCA)

PHQ-9: Patient Health Questionnaire-9

SGRQ: Saint George's Respiratory Questionnaire

\section{DATA AND ANALYSES}

\section{Comparison 1. Psychological therapies versus co-intervention}

\begin{tabular}{lllll}
\hline Outcome or subgroup title & No. of studies & $\begin{array}{l}\text { No. of partici- } \\
\text { pants }\end{array}$ & Statistical method & Effect size \\
\hline 1 Anxiety & 3 & 319 & Mean Difference (IV, Random, 95\% Cl) & $-4.41[-8.28,-0.53]$ \\
\hline 2 Quality of life & 2 & $\begin{array}{l}\text { Std. Mean Difference (IV, Random, } \\
95 \% \mathrm{Cl})\end{array}$ & Subtotals only \\
\hline \hline
\end{tabular}




\begin{tabular}{lllll}
\hline Outcome or subgroup title & No. of studies & $\begin{array}{l}\text { No. of partici- } \\
\text { pants }\end{array}$ & Statistical method & Effect size \\
\hline $\begin{array}{l}\text { 2.1 SGRQ and SF36 Physical } \\
\text { composite }\end{array}$ & 2 & 289 & $\begin{array}{l}\text { Std. Mean Difference (IV, Random, } \\
95 \% \text { Cl) }\end{array}$ & $-0.40[-0.88,0.08]$ \\
\hline $\begin{array}{l}\text { 2.2 SGRQ and SF36 Emo- } \\
\text { tional composite }\end{array}$ & 2 & 289 & $\begin{array}{l}\text { Std. Mean Difference (IV, Random, } \\
95 \% \text { Cl) }\end{array}$ & $-0.30[-1.03,0.44]$ \\
\hline $\begin{array}{l}\text { 3 Six minute walking dis- } \\
\text { tance }\end{array}$ & 2 & 268 & Mean Difference (IV, Fixed, 95\% Cl) & $-2.78[-58.49,52.94]$ \\
\hline
\end{tabular}

\section{Analysis 1.1. Comparison 1 Psychological therapies versus co-intervention, Outcome 1 Anxiety.}

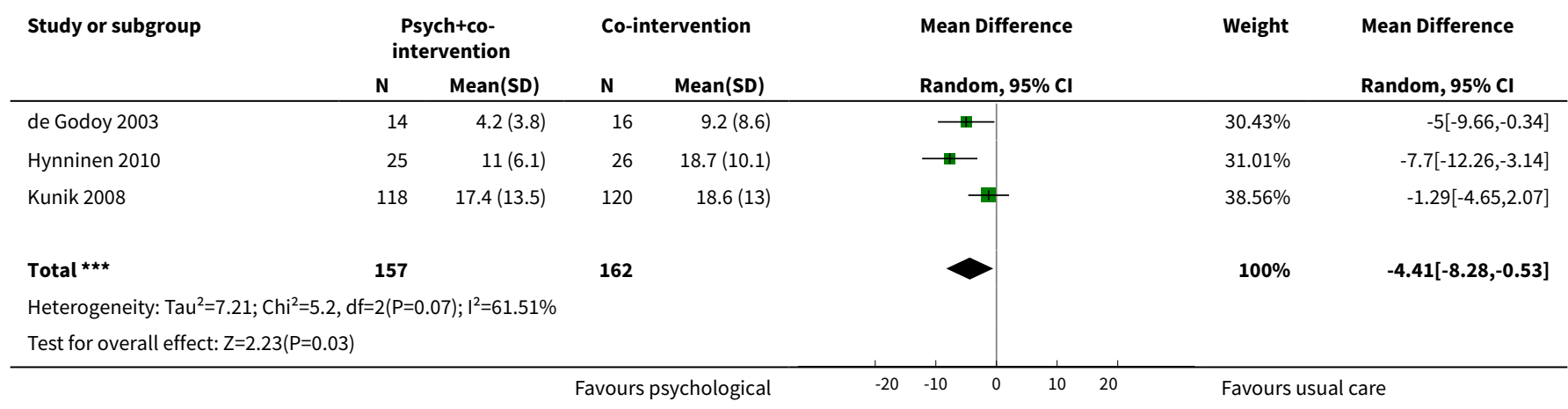

Analysis 1.2. Comparison 1 Psychological therapies versus co-intervention, Outcome 2 Quality of life.

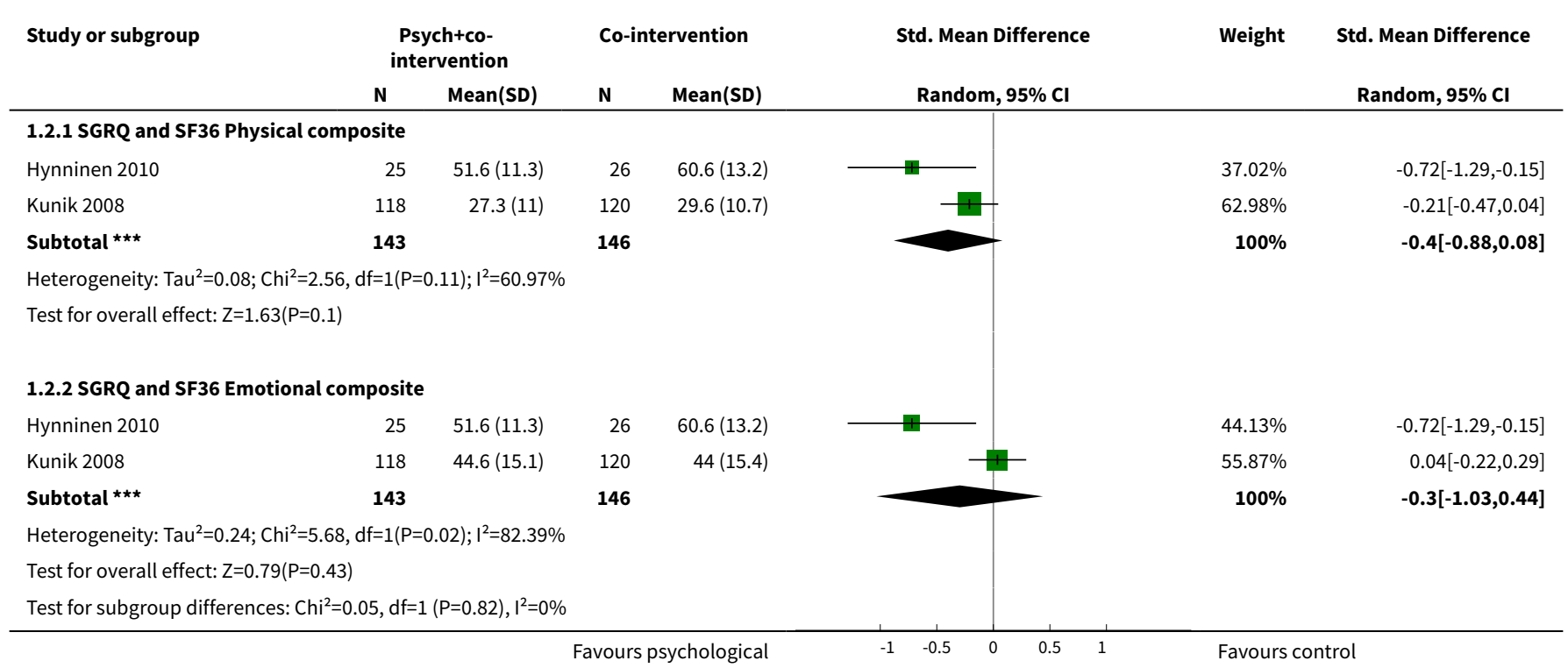


Analysis 1.3. Comparison 1 Psychological therapies versus co-intervention, Outcome 3 Six minute walking distance.

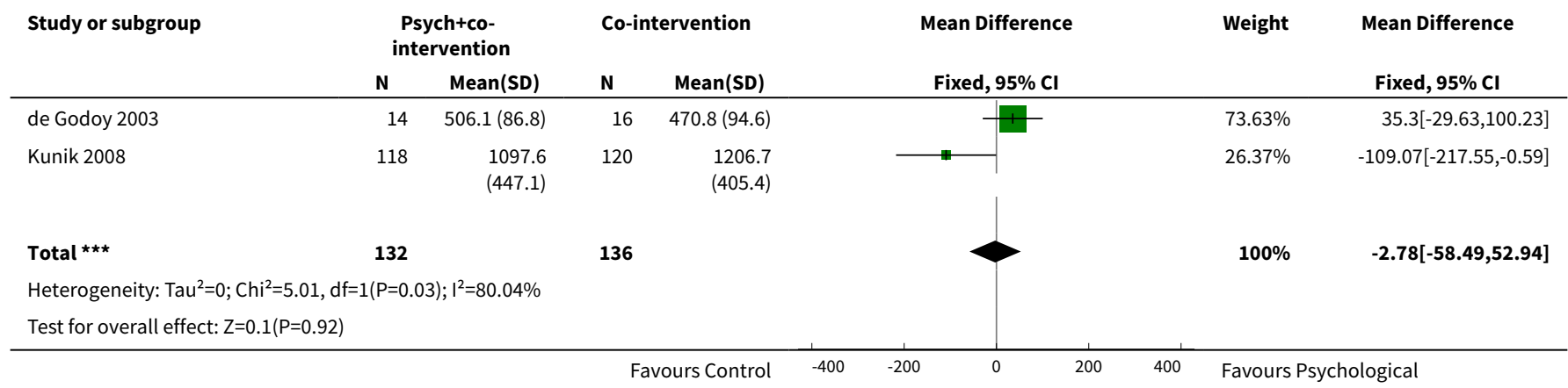

Comparison 2. Duration of intervention sub-group analyses - psychological therapies versus co-intervention

\begin{tabular}{|c|c|c|c|c|}
\hline Outcome or subgroup title & No. of studies & $\begin{array}{l}\text { No. of partici- } \\
\text { pants }\end{array}$ & Statistical method & Effect size \\
\hline 1 Anxiety & 3 & & $\begin{array}{l}\text { Mean Difference (IV, Random, 95\% } \\
\mathrm{CI})\end{array}$ & Subtotals only \\
\hline $\begin{array}{l}1.1 \text { Short-term ( } 0 \text { to } 3 \\
\text { months) }\end{array}$ & 3 & 319 & $\begin{array}{l}\text { Mean Difference (IV, Random, 95\% } \\
\mathrm{Cl} \text { ) }\end{array}$ & $-3.86[-6.63,-1.10]$ \\
\hline $\begin{array}{l}1.2 \text { Long term ( } 6 \text { to } 12 \\
\text { months) }\end{array}$ & 2 & 289 & $\begin{array}{l}\text { Mean Difference (IV, Random, 95\% } \\
\mathrm{Cl} \text { ) }\end{array}$ & $-4.30[-10.57,1.97]$ \\
\hline 2 Six minute walking distance & 2 & & $\begin{array}{l}\text { Mean Difference (IV, Random, 95\% } \\
\mathrm{Cl} \text { ) }\end{array}$ & Subtotals only \\
\hline $\begin{array}{l}2.1 \text { Short-term ( } 0 \text { to } 3 \\
\text { months) }\end{array}$ & 2 & 268 & $\begin{array}{l}\text { Mean Difference (IV, Random, 95\% } \\
\mathrm{Cl} \text { ) }\end{array}$ & $29.35[-30.13,88.82]$ \\
\hline $\begin{array}{l}2.2 \text { Long-term ( } 6 \text { to } 12 \\
\text { months) }\end{array}$ & 2 & 268 & $\begin{array}{l}\text { Mean Difference (IV, Random, 95\% } \\
\mathrm{Cl} \text { ) }\end{array}$ & $\begin{array}{l}-30.08[-170.92 \\
110.77]\end{array}$ \\
\hline $\begin{array}{l}3 \text { Quality of life - SGRQ and } \\
\text { SF36 Physical composite }\end{array}$ & 2 & & $\begin{array}{l}\text { Std. Mean Difference (IV, Random, } \\
95 \% \mathrm{Cl} \text { ) }\end{array}$ & Subtotals only \\
\hline $\begin{array}{l}3.1 \text { Short-term (0 to } 3 \\
\text { months) }\end{array}$ & 2 & 289 & $\begin{array}{l}\text { Std. Mean Difference (IV, Random, } \\
95 \% \mathrm{Cl} \text { ) }\end{array}$ & $-0.30[-0.73,0.13]$ \\
\hline $\begin{array}{l}3.2 \text { Long-term ( } 6 \text { to } 12 \\
\text { months) }\end{array}$ & 2 & 289 & $\begin{array}{l}\text { Std. Mean Difference (IV, Random, } \\
95 \% \mathrm{Cl} \text { ) }\end{array}$ & $-0.40[-0.88,0.08]$ \\
\hline $\begin{array}{l}4 \text { Quality of life - SGRQ and } \\
\text { SF36 Emotional composite }\end{array}$ & 2 & & $\begin{array}{l}\text { Std. Mean Difference (IV, Random, } \\
95 \% \mathrm{Cl} \text { ) }\end{array}$ & Subtotals only \\
\hline $\begin{array}{l}4.1 \text { Short-term ( } 0 \text { to } 3 \\
\text { months) }\end{array}$ & 2 & 289 & $\begin{array}{l}\text { Std. Mean Difference (IV, Random, } \\
95 \% \mathrm{Cl} \text { ) }\end{array}$ & $-0.17[-0.93,0.60]$ \\
\hline $\begin{array}{l}4.2 \text { Long-term ( } 6 \text { to } 12 \\
\text { months) }\end{array}$ & 2 & 289 & $\begin{array}{l}\text { Std. Mean Difference (IV, Random, } \\
95 \% \mathrm{CI} \text { ) }\end{array}$ & $-0.30[-1.03,0.44]$ \\
\hline
\end{tabular}


Analysis 2.1. Comparison 2 Duration of intervention sub-group analyses - psychological therapies versus co-intervention, Outcome 1 Anxiety.

\begin{tabular}{|c|c|c|c|c|c|c|c|}
\hline \multirow[t]{2}{*}{ Study or subgroup } & \multicolumn{2}{|c|}{$\begin{array}{l}\text { Psych+co- } \\
\text { intervention }\end{array}$} & \multicolumn{2}{|c|}{ Co-intervention } & \multirow{2}{*}{$\begin{array}{l}\text { Mean Difference } \\
\text { Random, } 95 \% \mathrm{Cl}\end{array}$} & \multirow[t]{2}{*}{ Weight } & \multirow{2}{*}{$\begin{array}{l}\text { Mean Difference } \\
\text { Random, } 95 \% \mathrm{Cl}\end{array}$} \\
\hline & $\mathbf{N}$ & $\operatorname{Mean}(S D)$ & $\mathbf{N}$ & Mean(SD) & & & \\
\hline \multicolumn{8}{|c|}{ 2.1.1 Short-term (0 to 3 months) } \\
\hline de Godoy 2003 & 14 & $4.2(3.8)$ & 16 & $9.2(8.6)$ & —- & $29.28 \%$ & $-5[-9.66,-0.34]$ \\
\hline Hynninen 2010 & 25 & $12.7(6.8)$ & 26 & $18.7(10)$ & $\longrightarrow$ & $29.1 \%$ & $-6[-10.68,-1.32]$ \\
\hline Kunik 2008 & 118 & $15.9(14.9)$ & 120 & $17.5(14.5)$ & +1 & $41.61 \%$ & $-1.57[-5.31,2.17]$ \\
\hline \multicolumn{8}{|c|}{ Heterogeneity: $\mathrm{Tau}^{2}=1.16 ; \mathrm{Chi}^{2}=2.47, \mathrm{df}=2(\mathrm{P}=0.29) ; \mathrm{I}^{2}=19.09 \%$} \\
\hline \multicolumn{8}{|c|}{ Test for overall effect: $\mathrm{Z}=2.74(\mathrm{P}=0.01)$} \\
\hline \multicolumn{8}{|c|}{ 2.1.2 Long term (6 to 12 months) } \\
\hline Hynninen 2010 & 25 & $11(6.1)$ & 26 & $18.7(10.1)$ & 1 & $47 \%$ & $-7.7[-12.26,-3.14]$ \\
\hline Kunik 2008 & 118 & $17.4(13.5)$ & 120 & $18.6(13)$ & & $53 \%$ & $-1.29[-4.65,2.07]$ \\
\hline \multicolumn{8}{|c|}{ Heterogeneity: $\mathrm{Tau}^{2}=16.37 ; \mathrm{Chi}^{2}=4.92, \mathrm{df}=1(\mathrm{P}=0.03) ; \mathrm{I}^{2}=79.66 \%$} \\
\hline \multicolumn{8}{|c|}{ Test for overall effect: $\mathrm{Z}=1.34(\mathrm{P}=0.18)$} \\
\hline Test for subgroup dif & $2, \mathrm{df}=$ & $P=0.9), I^{2}=0 \%$ & & & & & \\
\hline
\end{tabular}

Analysis 2.2. Comparison 2 Duration of intervention sub-group analyses psychological therapies versus co-intervention, Outcome 2 Six minute walking distance.

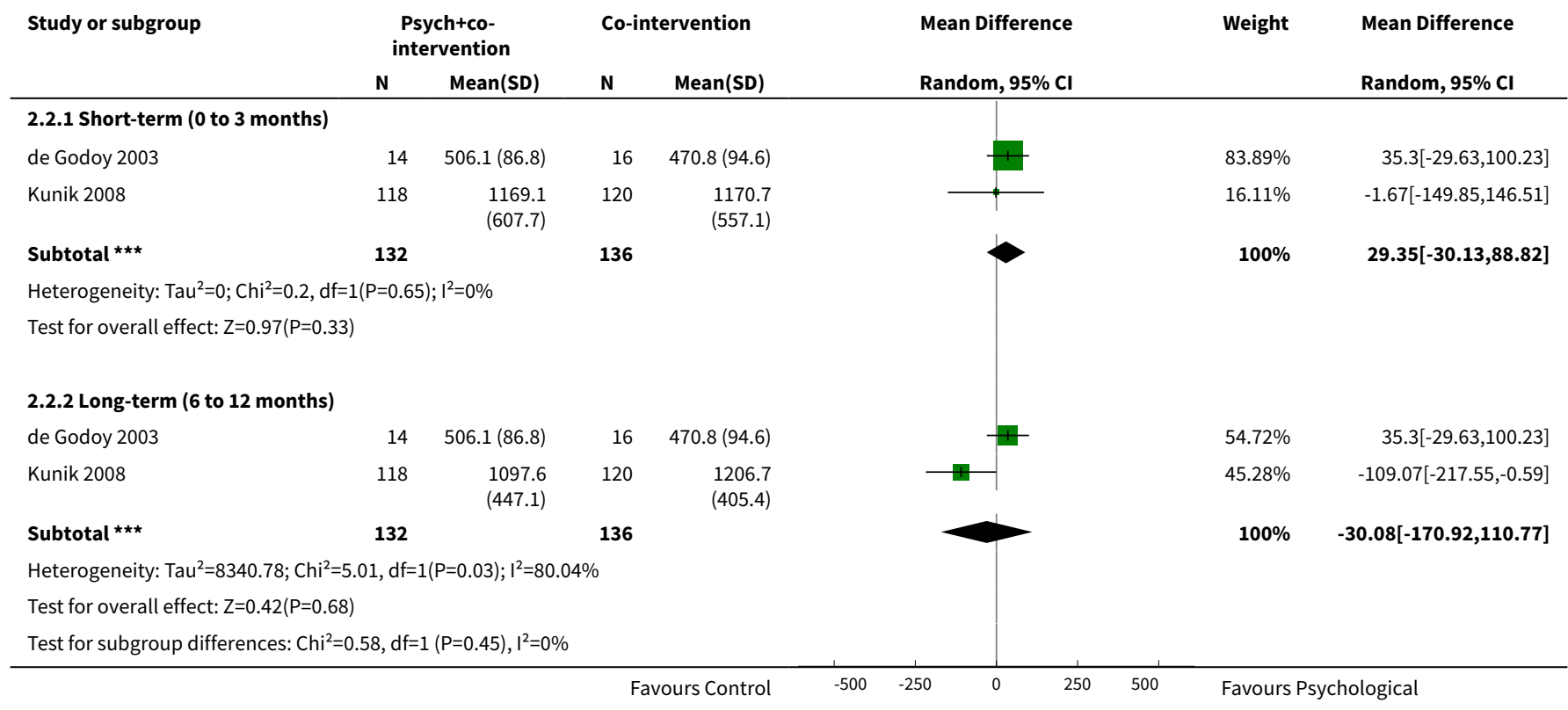


Analysis 2.3. Comparison 2 Duration of intervention sub-group analyses - psychological therapies versus co-intervention, Outcome 3 Quality of life - SGRQ and SF36 Physical composite.

\begin{tabular}{|c|c|c|c|c|c|c|c|}
\hline \multirow[t]{2}{*}{ Study or subgroup } & \multicolumn{2}{|c|}{$\begin{array}{l}\text { Psych+co- } \\
\text { intervention }\end{array}$} & \multicolumn{2}{|c|}{ Co-intervention } & \multirow{2}{*}{$\begin{array}{l}\text { Std. Mean Difference } \\
\text { Random, } 95 \% \mathrm{Cl}\end{array}$} & \multirow[t]{2}{*}{ Weight } & \multirow{2}{*}{$\begin{array}{l}\text { Std. Mean Difference } \\
\text { Random, } 95 \% \mathrm{CI}\end{array}$} \\
\hline & $\mathbf{N}$ & Mean(SD) & $\mathbf{N}$ & Mean(SD) & & & \\
\hline \multicolumn{8}{|c|}{ 2.3.1 Short-term ( 0 to 3 months) } \\
\hline Hynninen 2010 & 25 & $50.9(11.5)$ & 26 & $58.6(13.6)$ & -1 & $34.56 \%$ & $-0.6[-1.16,-0.04]$ \\
\hline Kunik 2008 & 118 & $28.1(12.2)$ & 120 & $29.8(11.8)$ & & $65.44 \%$ & $-0.14[-0.4,0.11]$ \\
\hline 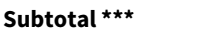 & 143 & & 146 & & & $100 \%$ & $-0.3[-0.73,0.13]$ \\
\hline \multicolumn{8}{|c|}{ Test for overall effect: $Z=1.37(P=0.17)$} \\
\hline \multicolumn{8}{|c|}{ 2.3.2 Long-term ( 6 to 12 months) } \\
\hline Hynninen 2010 & 25 & $51.6(11.3)$ & 26 & $60.6(13.2)$ & 世- & $37.02 \%$ & $-0.72[-1.29,-0.15]$ \\
\hline Kunik 2008 & 118 & $27.3(11)$ & 120 & $29.6(10.7)$ & & $62.98 \%$ & $-0.21[-0.47,0.04]$ \\
\hline Subtotal $\star \star \star ~$ & 143 & & 146 & & & $100 \%$ & $-0.4[-0.88,0.08]$ \\
\hline \multicolumn{8}{|c|}{ Test for overall effect: $Z=1.63(P=0.1)$} \\
\hline Test for subgroup dif & $09, \mathrm{df}=$ & $P=0.76), I^{2}=0 \%$ & & & & & \\
\hline
\end{tabular}

Analysis 2.4. Comparison 2 Duration of intervention sub-group analyses - psychological therapies versus co-intervention, Outcome 4 Quality of life - SGRQ and SF36 Emotional composite.

\begin{tabular}{|c|c|c|c|c|c|c|c|}
\hline \multirow[t]{2}{*}{ Study or subgroup } & \multicolumn{2}{|c|}{$\begin{array}{l}\text { Psych+co- } \\
\text { intervention }\end{array}$} & \multicolumn{2}{|c|}{ Co-intervention } & \multirow{2}{*}{$\begin{array}{l}\text { Std. Mean Difference } \\
\text { Random, } 95 \% \mathrm{Cl} \\
\end{array}$} & \multirow[t]{2}{*}{ Weight } & \multirow{2}{*}{$\begin{array}{l}\text { Std. Mean Difference } \\
\text { Random, } 95 \% \mathrm{Cl}\end{array}$} \\
\hline & $\mathbf{N}$ & Mean(SD) & $\mathbf{N}$ & Mean(SD) & & & \\
\hline \multicolumn{8}{|c|}{ 2.4.1 Short-term ( 0 to 3 months) } \\
\hline Hynninen 2010 & 25 & $50.9(11.5)$ & 26 & $58.6(13.6)$ & $\longrightarrow$ & $44.72 \%$ & $-0.6[-1.16,-0.04]$ \\
\hline Kunik 2008 & 118 & $46.6(15.1)$ & 120 & $43.8(14.7)$ & & $55.28 \%$ & $0.19[-0.07,0.44]$ \\
\hline Subtotal $* \star \star$ & 143 & & 146 & & & $100 \%$ & $-0.17[-0.93,0.6]$ \\
\hline \multicolumn{8}{|c|}{ Heterogeneity: $\mathrm{Tau}^{2}=0.26 ; \mathrm{Chi}^{2}=6.25, \mathrm{df}=1(\mathrm{P}=0.01) ; \mathrm{I}^{2}=84 \%$} \\
\hline \multicolumn{8}{|c|}{ Test for overall effect: $\mathrm{Z}=0.42(\mathrm{P}=0.67)$} \\
\hline \multicolumn{8}{|c|}{ 2.4.2 Long-term ( 6 to 12 months) } \\
\hline Hynninen 2010 & 25 & $51.6(11.3)$ & 26 & $60.6(13.2)$ & —- & $44.12 \%$ & $-0.72[-1.29,-0.15]$ \\
\hline Kunik 2008 & 118 & $44.6(15.8)$ & 120 & $44(15.4)$ & & $55.88 \%$ & $0.04[-0.22,0.29]$ \\
\hline 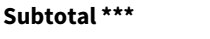 & 143 & & 146 & & & $100 \%$ & $-0.3[-1.03,0.44]$ \\
\hline \multicolumn{8}{|c|}{ Test for overall effect: $Z=0.79(P=0.43)$} \\
\hline Test for subgroup dif & $06, d f=$ & $P=0.81), I^{2}=0 \%$ & & & & & \\
\hline
\end{tabular}

\section{APPENDICES}

\section{Appendix 1. CCMDCTR - core MEDLINE search}

Core search strategy used to inform Cochrane Common Mental Disorders' specialised register: OVID Medline

A weekly search alert based on condition + RCT filter only

1. [MeSH Headings]: 
eating disorders/ or anorexia nervosa/ or binge-eating disorder/ or bulimia nervosa/ or female athlete triad syndrome/ or pica/ or hyperphagia/ or bulimia/ or self-injurious behavior/ or self mutilation/ or suicide/ or suicidal ideation/ or suicide, attempted/ or mood disorders/ or affective disorders, psychotic/ or bipolar disorder/ or cyclothymic disorder/ or depressive disorder/ or depression, postpartum/ or depressive disorder, major/ or depressive disorder, treatment-resistant/ or dysthymic disorder/ or seasonal affective disorder/ or neurotic disorders/ or depression/ or adjustment disorders/ or exp antidepressive agents/ or anxiety disorders/ or agoraphobia/ or neurocirculatory asthenia/ or obsessive-compulsive disorder/ or obsessive hoarding/ or panic disorder/ or phobic disorders/ or stress disorders, traumatic/ or combat disorders/ or stress disorders, post-traumatic/ or stress disorders, traumatic, acute/ or anxiety/ or anxiety, castration/ or koro/ or anxiety, separation/ or panic/or exp anti-anxiety agents/ or somatoform disorders/ or body dysmorphic disorders/ or conversion disorder/ or hypochondriasis/or neurasthenia/ or hysteria/ or munchausen syndrome by proxy/ or munchausen syndrome/ or fatigue syndrome, chronic/ or obsessive behavior/ or compulsive behavior/ or behavior, addictive/ or impulse control disorders/ or firesetting behavior/ or gambling/ or trichotillomania/ or stress, psychological/ or burnout, professional/ or sexual dysfunctions, psychological/ or vaginismus/ or Anhedonia/ or Affective Symptoms/ or *Mental Disorders/

\section{2. [Title/Author Keywords]:}

(eating disorder ${ }^{\star}$ or anorexia nervosa or bulimi ${ }^{\star}$ or binge eat ${ }^{\star}$ or (self adj (injur ${ }^{\star}$ or mutilat $^{\star}$ )) or suicide* or suicidal or parasuicid ${ }^{\star}$ or mood disorder* or affective disorder ${ }^{\star}$ or bipolar i or bipolar ii or (bipolar and (affective or disorder $\left.{ }^{\star}\right)$ ) or mania or manic or cyclothymic ${ }^{\star}$ or depression or depressive or dysthymi ${ }^{\star}$ or neurotic or neurosis or adjustment disorder* or antidepress* or anxiety disorder ${ }^{\star}$ or agoraphobia or obsess ${ }^{\star}$ or compulsi ${ }^{\star}$ or panic or phobi ${ }^{\star}$ or ptsd or posttrauma ${ }^{\star}$ or post trauma* or combat or somatoform or somati\#ation or medical ${ }^{\star}$ unexplained or body dysmorphi* or conversion disorder or hypochondria* or neurastheni* or hysteria or munchausen or chronic fatigue* or gambling or trichotillomania or vaginismus or anhedoni ${ }^{\star}$ or affective symptoms or mental disorder* or mental health).ti,kf.

\section{3. [RCT filter]:}

(controlled clinical trial.pt. or randomised controlled trial.pt. or (randomi\#ed or randomi\#ation).ab,ti. or randomly.ab. or (random adj3

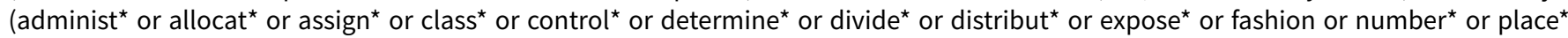
or recruit ${ }^{\star}$ or subsitut ${ }^{\star}$ or treat*)).ab. or placebo*.ab,ti. or drug therapy.fs. or trial.ab,ti. or groups.ab. or (control* adj3 (trial* or study or studies)).ab,ti. or ((singl* or doubl* or tripl ${ }^{\star}$ or trebl*) adj3 (blind ${ }^{\star}$ or mask ${ }^{\star}$ or dummy*)).mp. or clinical trial, phase ii/ or clinical trial, phase iii/ or clinical trial, phase iv/ or randomised controlled trial/ or pragmatic clinical trial/ or (quasi adj (experimental or random)).ti,ab. or

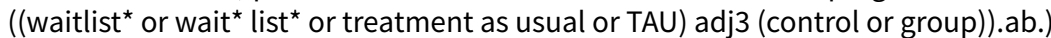

\section{4. (1 and 2 and 3 )}

Records are screened for reports of RCTs within the scope of the Cochrane Common Mental Disorders Group. Secondary reports of RCTs are tagged to the appropriate study record.

Similar weekly search alerts are also conducted on OVID EMBASE and PsycINFO, using relevant subject headings (controlled vocabularies) and search syntax, appropriate to each resource.

\section{Appendix 2. Additional search strategies (CENTRAL and PsycINFO):}

\section{CENTRAL search strategy:}

Issue 8, 2013, $n=124$

\#1. MeSH descriptor LUNG DISEASES, OBSTRUCTIVE, this term only

\#2. MeSH descriptor PULMONARY DISEASE, CHRONIC OBSTRUCTIVE explode all trees

\#3. emphysema*

\#4. chronic ${ }^{\star}$ near/3 bronchiti*

\#5. (obstruct ${ }^{\star}$ ) near/3 (pulmonary or lung* or airway* or airflow* or bronch $^{\star}$ or respirat ${ }^{\star}$ )

\#6. (COPD or COAD or COBD or AECB)

\#7. (\#1 OR \#2 OR \#3 OR \#4 OR \#5 OR \#6)

\#8. MeSH descriptor ANXIETY, this term only

\#9. MeSH descriptor ANXIETY DISORDERS explode all trees

\#10. MeSH descriptor ANXIETY, SEPARATION, this term only

\#11. MeSH descriptor PANIC, this term only

\#12. MeSH descriptor OBSESSIVE BEHAVIOR explode all trees

\#13. MeSH descriptor COMPULSIVE BEHAVIOR explode all trees

\#14. MeSH descriptor STRESS, PSYCHOLOGICAL explode all trees

\#15. MeSH descriptor NEUROTIC DISORDERS, this term only

\#16. (anxiety or phobi* or agoraphobi* or claustrophobi* or PTSD or post-trauma* or posttrauma or (post NEXT trauma*) or (combat NEXT disorder) or panic or OCD or obsess ${ }^{\star}$ or compulsi ${ }^{\star}$ or GAD or stress ${ }^{\star}$ or distress ${ }^{\star}$ or neurosis or neuroses or neurotic or psychoneuro*)

\#17. (\#8 OR \#9 OR \#10 OR \#11 OR \#12 OR \#13 OR \#14 OR \#15 OR \#16)

\#18. (\#7 and \#17)

\#19. (SR-DEPRESSN OR HS-DEPRESSN)

\#20. (SR-AIRWAYS OR HS-AIRWAYS)

\#21. \#19 OR \#20

Psychological therapies for the treatment of anxiety disorders in chronic obstructive pulmonary disease (Review)

Copyright (c) 2017 The Cochrane Collaboration. Published by John Wiley \& Sons, Ltd. 
\#22. (\#18 NOT \#21)

\section{OVID PsycINFO search strategy:}

Searched 26 September 2013, $n=98$

1. Lung Disorders/

2. exp Chronic Obstructive Pulmonary Disease/

3. (chronic $^{\star}$ adj3 bronchiti $\left.{ }^{\star}\right) \cdot \mathrm{mp}$.

4. emphysema*.mp.

5. (obstruct* adj3 (pulmonary or lung* or airway* or airflow* or bronch $^{\star}$ or respirat $^{\star}$ )).mp.

6. (COPD or COAD or COBD or AECB).mp.

7. or/1-6

8. $\exp$ Anxiety/

9. $\exp$ Anxiety Disorders/

10.exp Phobias/

11.exp Neurosis/

12.exp Stress/

13.exp Trauma/

14.Panic Attack/ or Panic/ or Panic Disorder/

15.exp Fear/

16. (anxiety or phobi ${ }^{\star}$ or agoraphobi ${ }^{\star}$ or claustrophobi ${ }^{\star}$ or PTSD or post-trauma* or posttrauma or post trauma* or combat disorder or panic or OCD or obsess ${ }^{\star}$ or compulsi ${ }^{\star}$ or GAD or stress ${ }^{\star}$ or distress ${ }^{\star}$ or neurosis or neuroses or neurotic or psychoneuro $\left.{ }^{\star}\right) . \mathrm{mp}$.

$17.0 \mathrm{r} / 8-16$

18.treatment effectiveness evaluation.sh.

19.clinical trials.sh.

20.mental health program evaluation.sh.

21.placebo.sh.

22. placebo*.ti,ab.

23.randomly.ab.

24.randomi\#ed.ti,ab.

25.trial*.ti,ab.

26. ((singl ${ }^{\star}$ or doubl $^{\star}$ or trebl ${ }^{\star}$ or tripl $\left.{ }^{\star}\right)$ adj3 (blind ${ }^{\star}$ or mask $^{\star}$ or dummy)).mp.

27. (control ${ }^{\star}$ adj3 (trial ${ }^{\star}$ or study or studies or group $\left.\left.{ }^{\star}\right)\right)$. ti,ab.

28."2000".md.

29. factorial*.ti,ab.

30. allocat ${ }^{\star}$.ti,ab.

31.assign*.ti,ab.

32. volunteer ${ }^{\star}$.ti,ab.

33. (crossover\$ or cross over $\left.{ }^{\star}\right)$.ti,ab.

34. (quasi adj (experimental or random $\left.{ }^{\star}\right)$ ).mp.

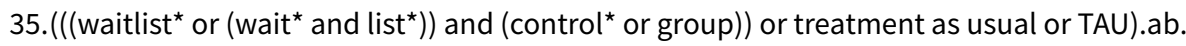

36.or/18-35

37.(7 and 17 and 36)

\section{CONTRIBUTIONS OF AUTHORS}

Protocol prepared by Zafar A Usmani, Kristin V Carson and Karen Heslop-Marshall with feedback provided by Adrian J Easterman, Anthony De Soyza and Brian J Smith.

Data screened and extracted by ZU, KC and HM, data entry, analysis and manuscript preparation by ZU and KC with feedback on manuscript by $A E, A D$ and $B S$.

\section{DECLARATIONS OF INTEREST}

Dr Zafar Usmani has previously received a grant from Cochrane Airways for his Cochrane Review. He has not received any other funding for his research related to the management of anxiety in people with COPD.

Psychological therapies for the treatment of anxiety disorders in chronic obstructive pulmonary disease (Review) 
Kristin Carson has received travel grants from the Thoracic Society of Australia and New Zealand, Healthy Development Adelaide (associated with The University of Adelaide) and the Young Professionals Group (associated with SA Health) to attend national and international conferences. She has received financial support and grants from multiple organisations in the past year including the Australian and New Zealand School of Government, the National Health and Medical Research Council, Cancer Australia, the Thoracic Society of Australia and New Zealand and Seeley International, toward supporting several research initiatives unrelated to this particular Cochrane Review.

Karen Heslop-Marshall has received fees for speaker meetings or consultancy work on management of anxiety and depression in COPD from a variety of commercial companies and has received a NIHR Fellowship grant to undertake a RCT of CBT in COPD.

Dr De Soyza has received no fees nor grants that relate to anxiety and depression management in COPD. He has received fees for speaker meetings or consultancy work on management of COPD airways disease management from a variety of commercial companies. He has also received financial support from multiple partners in the past to attend national congresses/symposia and has also had co-funding offers towards a multi-centre bronchiectasis grant.

Professor Brian Smith has received grant funding in the past year from the Australian and New Zealand School of Government, the National Health and Medical Research Council, Cancer Australia, the Thoracic Society of Australia and New Zealand and Seeley International, toward supporting several research initiatives unrelated to this particular Cochrane Review.

\section{SOURCES OF SUPPORT}

\section{Internal sources}

- Respiratory Department - The Queen Elizabeth Hospital, Australia.

\section{External sources}

- No sources of support supplied

\section{DIFFERENCES BETWEEN PROTOCOL AND REVIEW}

We did not conduct sensitivity analyses due to the small number of included studies reported within each meta-analysis.

\section{N DEX TERMS}

\section{Medical Subject Headings (MeSH)}

Anxiety Disorders [*therapy]; Bronchitis, Chronic [psychology]; Emphysema [psychology]; Exercise Tolerance; Psychotherapy [ ${ }^{\star}$ methods]; Pulmonary Disease, Chronic Obstructive [ ${ }^{*}$ psychology]; Quality of Life; Randomized Controlled Trials as Topic

\section{MeSH check words}

Female; Humans; Male 